\title{
Relative computability and uniform continuity of relations
}

\author{
ARNO PAULY \\ MARTIN ZIEGLER
}

\begin{abstract}
A type-2 computable real function is necessarily continuous; and this remains true for computations relative to any oracle. Conversely, by the Weierstrass Approximation Theorem, every continuous $f:[0 ; 1] \rightarrow \mathbb{R}$ is computable relative to some oracle.

In their search for a similar topological characterization of relatively computable multi-valued functions $f:[0 ; 1] \rightrightarrows \mathbb{R}$ (also known as multi-functions or relations), Brattka and Hertling (1994) have considered two notions: weak continuity (which is weaker than relative computability) and strong continuity (which is stronger than relative computability). Observing that uniform continuity plays a crucial role in the Weierstrass Theorem, we propose and compare several notions of uniform continuity for relations. Here, due to the additional quantification over values $y \in f(x)$, new ways arise of (linearly) ordering quantifiers-yet none turns out as satisfactory.

We are thus led to a concept of uniform continuity based on the Henkin quantifier; and prove it necessary for relative computability of compact real relations. In fact iterating this condition yields a strict hierarchy of notions each necessary - and the $\omega$-th level also sufficient - for relative computability. A refined, quantitative analysis exhibits a similar topological characterization of relative polynomial-time computability.
\end{abstract}

2010 Mathematics Subject Classification 03D78 (primary); 03C80 (secondary)

Keywords: multivaluedness, uniform continuity, Henkin Quantifier

\section{Introduction}

A simple counting argument shows that not every (total) integer function $f: \mathbb{N} \rightarrow \mathbb{N}=$ $\{1,2, \ldots\}$ can be computable; on the other hand, each such function can be encoded into an oracle $\mathcal{O} \subseteq\{0,1\}^{*}$ that renders it computable relative to $\mathcal{O}$. Over real numbers, similarly, not every total $f:[0 ; 1] \rightarrow \mathbb{R}$ can be computable for cardinality reasons; 
and this remains true for oracle machines. In fact it is well-known that any function $f$ computably mapping approximations of real numbers $x$ to approximations of $f(x)$ must necessarily be continuous*; and the same remains true for oracle computations. Even more surprisingly, this implication can be reversed: If a (say, real) function $f$ is continuous, then there exists an oracle which renders $f$ computable ${ }^{\dagger}$. This can for instance be concluded from the Weierstrass Approximation Theorem. A far reaching generalization from the reals to so-called admissibly represented spaces is the KreitzWeihrauch Theorem, cf. e.g. WEIHRAUCH [28, THEOREM 3.2.11] and SCHRÖDER [24] and compare the Myhill-Shepherdson Theorem in Domain Theory. The equivalence between continuity and relative computability has led DANA SCOTT to regard continuity as an approximation to computability.

Now for structural reasons, multivalued mappings arise naturally in Computable Analysis as building blocks to (even single-valued) functions [2]. Moreover many computational problems themselves are more naturally expressed as relations (i.e. multivalued) rather than as functions. For instance when diagonalizing a given real symmetric matrix, one is interested in some basis of eigenvectors, not a specific one [8]; likewise, for solving a bimatrix game, there may be various Nash equilibria to pick from [21]. It is thus natural to consider computations which, given (some encoding of) $x$, intensionally choose and output some value $y \in f(x)$. Indeed, a multifunction may well be computable yet admit no continuous single-valued selection; cf. e.g. WEIHRAUCH [28, EXERCISE 5.1.13] or LUCKHARDT [18]. Hence multivaluedness avoids some of the topological restrictions of single-valued functions-but not all of them: for instance the sign relation $0<x \mapsto\{1\}$, $0>x \mapsto\{-1\}, 0 \mapsto[-1 ; 1]$ remains uncomputable. More generally it is known that a multifunction $f$ admits an oracle rendering it computable (i.e. is relatively computable) iff $f$ has a continuous so-called realizer, that is a function mapping any infinite binary string encoding some $x$ to an infinite binary string encoding some $y \in f(x)$.

Now the single-valued case raises the hope for a topological characterization of relative computability also for multivalued functions: intrinsical, i.e. in the language of metric spaces rather than in the language of represented spaces. Such an investigation has been pursued by BRATTKA and HERTLING [7], yielding both necessary and sufficient conditions for a relation to be relatively computable (which, there, is called relative continuity ${ }^{\ddagger}$ ). BRATTKA and HERTLING have established what remains to-date the best counterpart to the Kreitz-Weihrauch Theorem for the multivalued case:

\footnotetext{
*In particular the sign function $0<x \mapsto 1,0>x \mapsto-1,0 \mapsto 0$ is uncomputable.

${ }^{\dagger}$ It has been observed by J. MILLER that a continuous function $f:[0 ; 1] \rightarrow[0 ; 1]$ will usually not have a least oracle rendering it computable [19]

${ }^{\ddagger}$ We have two reasons for changing the terminology. First, the relative here can only refer to the representation, however, the first author strongly feels that a representation should be
} 
Fact 1.1 Let $X, Y$ be separable metric spaces and $Y$ in addition complete. Then a pointwise closed relation $f: X \rightrightarrows Y$ is relatively computable iff it has a strongly continuous tightening ${ }^{\S}$.

Here, being pointwise closed means that $f(x):=\{y \in Y:(x, y) \in f\}$ is a closed subset for every $x \in X$. We shall freely switch between the viewpoint of $f: \subseteq X \rightrightarrows Y$ being a relation $(f \subseteq X \times Y)$ and being a set-valued partial mapping $f: \subseteq X \rightarrow 2^{Y}, x \mapsto f(x)$. Such $f$ is considered total (written $f: X \rightrightarrows Y$ ) if $\operatorname{dom}(f):=\{x \in X: f(x) \neq \emptyset\}$ coincides with $X$. Following WeIHRAUCh [30, Definition 7], $g$ is said to tighten $f$ (and $f$ to loosen $g$ ) if both $\operatorname{dom}(f) \subseteq \operatorname{dom}(g)$ and $\forall x \in \operatorname{dom}(f): g(x) \subseteq f(x)$ hold; see Figure 1a) and observe that tightening is reflexive and transitive. Furthermore write $f[S]:=\bigcup_{x \in S} f(x)$ for $S \subseteq X$ and $\operatorname{range}(f):=f[X]$; also $\left.f\right|_{S}:=f \cap(S \times Y)$ and $\left.f\right|^{T}:=f \cap(X \times T)$ for $T \subseteq Y$. Finally let $f^{-1}:=\{(y, x):(x, y) \in f\}$ denote the inverse of $f$, i.e. such that $\left(f^{-1}\right)^{-1}=f$ and $\operatorname{range}(f)=\operatorname{dom}\left(f^{-1}\right)$.
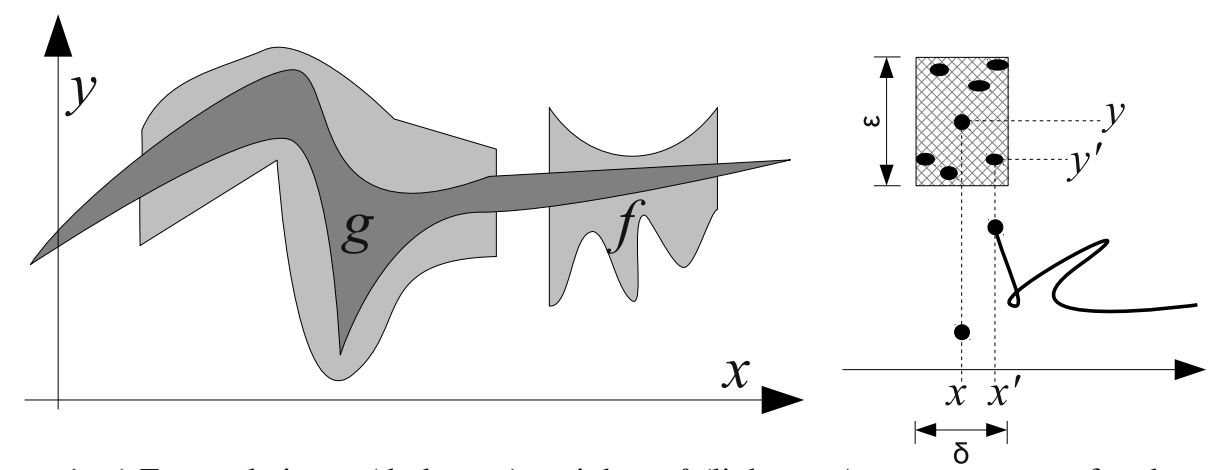

Figure 1: a) For a relation $g$ (dark gray) to tighten $f$ (light gray) means no more freedom (yet the possibility) to choose some $y \in g(x)$ than to choose some $y \in f(x)$ (whenever possible). b) Illustrating $\epsilon-\delta$-continuity in $(x, y)$ for a relation (black)

\subsection{Overview}

Section 2 recalls the notions of weak and strong, and introduces new variants of, continuity for multivalued functions (Definition 2.1) and their relations to relative computability (Section 2.1). These notions arise from the classical $\varepsilon-\delta$ definition with

considered as basic a property of a space than say a topology (cf. [22]). Second, by reserving the term continuous for notions expressed in the language of metric spaces only, our goal of finding such a counterpart to relatively computable for multivalued functions becomes clearer.

${ }^{\S}$ We reserve the original term "restriction" to denote either $\left.f\right|_{A}:=f \cap(A \times Y)$ or $\left.f\right|^{B}:=$ $f \cap(X \times B)$ for some $A \subseteq X$ or $B \subseteq Y$. 
additional, universal and/or existential quantification over the set-values. However all linear placements of the quantifiers turn out as either too weak or too strong. In Section 3 we therefore employ the (partially ordered) Henkin quantifier to explore a notion of uniform continuity (Definition 3.1) already suggested by M.J. BEESON. Section 4 iterates this notion to yield a strict hierarchy (Example 4.2); and our main result (Theorem 4.10) proves its $\omega$-th level equivalent to relative computability. We finally refine this topological characterization to relatively polynomial-time computable relations (Corollary 6.5).

\section{Continuity for Relations}

For multivalued mappings, the literature knows a variety of easily confusable notions of continuity; see for instance KLEIN and THOMPSON [12, §7] or SCHODL and NEUMAIER [23]. Some of them capture the intuition that, upon input $x$, all $y \in f(x)$ occur as output for some 'nondeterministic' choice [3, SECTION 7]; or that the 'value' $f(x)$ be produced extensionally as a set [26]. We pursue here the original conception that, on input $x$, some value $y \in f(x)$ be output.

Definition 2.1 Let $(X, d)$ and $(Y, e)$ denote metric spaces and abbreviate $B(x, r):=$ $\left\{x^{\prime} \in X: d\left(x, x^{\prime}\right)<r\right\} \subseteq X$ and $\bar{B}(x, r):=\left\{x^{\prime} \in X: d\left(x, x^{\prime}\right) \leq r\right\}$; similarly for $Y$. Now fix some $f: \subseteq X \rightrightarrows Y$ and call $(x, y) \in f$ a point of continuity of $f$ if the following formula holds (cf. Figure $1 b$ ):

$$
\forall \varepsilon>0 \quad \exists \delta>0 \quad \forall x^{\prime} \in B(x, \delta) \cap \operatorname{dom}(f) \exists y^{\prime} \in B(y, \varepsilon) \cap f\left(x^{\prime}\right) .
$$

a) Call $f$ strongly continuous if every $(x, y) \in f$ is a point of continuity of $f$ :

$$
\forall x \in \operatorname{dom}(f) \forall y \in f(x) \quad \forall \varepsilon>0 \quad \exists \delta>0 \quad \forall x^{\prime} \in B(x, \delta) \cap \operatorname{dom}(f) \quad \exists y^{\prime} \in B(y, \varepsilon) \cap f\left(x^{\prime}\right) .
$$

b) Call $f$ weakly continuous if the following holds:

$$
\forall x \in \operatorname{dom}(f) \quad \exists y \in f(x) \quad \forall \varepsilon>0 \quad \exists \delta>0 \quad \forall x^{\prime} \in B(x, \delta) \cap \operatorname{dom}(f) \quad \exists y^{\prime} \in B(y, \varepsilon) \cap f\left(x^{\prime}\right) .
$$

c) Call $f$ semi-uniformly weakly continuous if the following holds:

$$
\forall \varepsilon>0 \exists \delta>0 \quad \forall x \in \operatorname{dom}(f) \quad \exists y \in f(x) \forall x^{\prime} \in B(x, \delta) \cap \operatorname{dom}(f) \quad \exists y^{\prime} \in B(y, \varepsilon) \cap f\left(x^{\prime}\right) .
$$

d) Call $f$ nonuniformly weakly continuous if the following holds:

$$
\forall \varepsilon>0 \quad \forall x \in \operatorname{dom}(f) \quad \exists \delta>0 \quad \exists y \in f(x) \quad \forall x^{\prime} \in B(x, \delta) \cap \operatorname{dom}(f) \quad \exists y^{\prime} \in B(y, \varepsilon) \cap f\left(x^{\prime}\right) .
$$

e) Call $f$ uniformly strongly continuous if the following holds:

$$
\forall \varepsilon>0 \exists \delta>0 \quad \forall x \in \operatorname{dom}(f) \quad \forall y \in f(x) \forall x^{\prime} \in B(x, \delta) \cap \operatorname{dom}(f) \quad \exists y^{\prime} \in B(y, \varepsilon) \cap f\left(x^{\prime}\right) .
$$


f) Call $f$ hemi-uniformly strongly continuous if the following holds:

$$
\forall \varepsilon>0 \quad \forall x \in \operatorname{dom}(f) \exists \delta>0 \quad \forall y \in f(x) \forall x^{\prime} \in B(x, \delta) \cap \operatorname{dom}(f) \quad \exists y^{\prime} \in B(y, \varepsilon) \cap f\left(x^{\prime}\right) .
$$
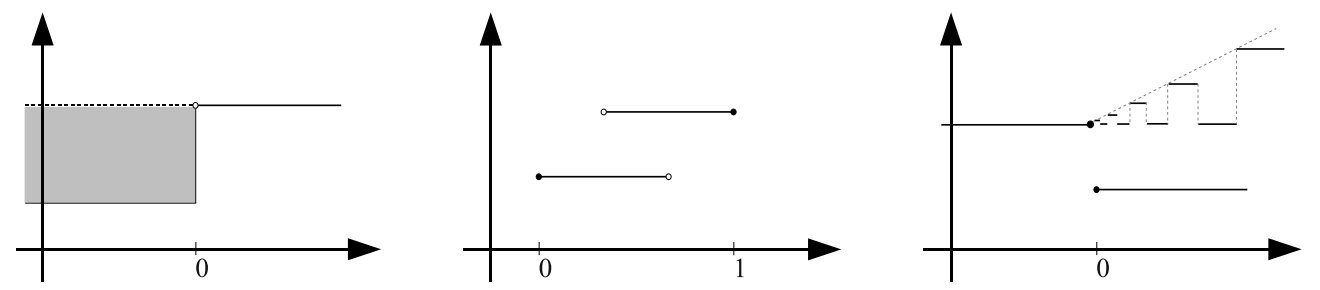

Figure 2: a) Example of a semi-uniformly weakly continuous but not weakly continuous relation. b) A hemi-uniformly strongly continuous relation which is not uniformly strongly continuous. c) A compact, weakly and semi-uniformly weakly continuous relation which is not computable relative to any oracle.

Items a) and b) are quoted from [7, Definition 2.1]; In the single-valued case, quantifications over $y \in f(x)$ and $y^{\prime} \in f\left(x^{\prime}\right)$ cancel: all a),b),d),f) collapse to classical continuity; and both c) and e) to uniform continuity. In the multivalued case, however, these notions are easily seen distinct. Moreover in the single-valued case it is well-known that the set of points of continuity can be $\mathrm{G}_{\delta}$ but not higher in the Borel hierarchy - a fact that GREGORIADES [10] has generalized to multivalued functions. Note that, in $\mathrm{f}$ ), $\delta$ may depend on $x$ but not on $y$; whereas $y$ may depend on $\varepsilon$ in c) but not in $\mathrm{b}$ ). We collect some logical connections between the various notions:

\section{Lemma $2.2 \quad$ a) Strong continuity implies weak continuity}

b) but not vice versa.

c) Weak continuity implies nonuniform weak continuity.

d) Semi-uniform weak continuity implies nonuniform weak continuity.

e) Let $f$ be semi-uniformly weakly continuous and suppose that $f(x) \subseteq Y$ is compact for every $x \in X$. Then $f$ is weakly continuous.

f) Uniform strong continuity implies hemi-uniform strong continuity which in turn implies strong continuity.

g) For compact $\operatorname{dom}(f) \subseteq X$, nonuniform weak continuity implies semi-uniform weak continuity.

h) If $f(x) \subseteq Y$ is compact for every $x \in X$, then strong continuity implies hemi-uniform strong continuity. 
j) If $f \subseteq X \times Y$ is compact and strongly continuous, it is uniformly strongly continuous.

k) If $f \subseteq X \times Y$ is compact, then so are $\operatorname{dom}(f) \subseteq X$ and $f[S] \subseteq Y$, for every closed $S \subseteq X$; in particular $f(x)$ is compact.

$\ell)$ If $X$ is compact and single-valued total $f: X \rightarrow Y$ is continuous, then both $f \subseteq X \times Y$ and its inverse $f^{-1} \subseteq Y \times X$ are compact.

Note that the (classically trivial) implication from (weak semi-) uniform continuity to (weak) continuity in e) is based on the (again, classically trivial) hypothesis that $f(x) \subseteq Y$ be compact. Similarly, the classical fact that continuity on a compact set classically yields uniform continuity is generalized in $\mathrm{g})+\mathrm{j}$ ).

Proof Items a),c), d), and f) are obvious.

b) is due to [7, Proposition 2.3]; compare Example 2.3d).

e) Fix $x \in \operatorname{dom}(f)$. By hypothesis there exists, to every $\varepsilon=1 / n$, some $\delta_{n}$ and $y_{n} \in f(x)$ with: $\forall x^{\prime} \in B\left(x, \delta_{n}\right) \cap \operatorname{dom}(f) \exists y^{\prime} \in B\left(y_{n}, 1 / n\right) \cap f\left(x^{\prime}\right)$. Now since $f(x)$ is compact, some subsequence $y_{n_{m}}$ of $y_{n}$ converges to, say, $y_{0} \in f(x)$ with $d\left(y_{n_{m}}, y_{0}\right) \leq 1 / m$. We claim that this $y_{0}$ (which does not depend on $\varepsilon$ anymore) satisfies

$$
\forall \varepsilon=2 / m>0 \quad \exists \delta:=\delta_{n_{m}}>0 \quad \forall x^{\prime} \in B(x, \delta) \cap \operatorname{dom}(f) \quad \exists y^{\prime} \in B\left(y_{0}, \varepsilon\right) \cap f\left(x^{\prime}\right) .
$$

Indeed, to arbitrary $x^{\prime} \in B\left(x, \delta_{n_{m}}\right) \cap \operatorname{dom}(f)$, the hypothesis yields some $y^{\prime} \in$ $B\left(y_{n_{m}}, 1 / m\right) \cap f\left(x^{\prime}\right)$. Then, by the triangle inequality, it follows that $y^{\prime} \in$ $B\left(y_{0}, 2 / m\right)$.

Note that a different $x$ may require a different subsequence $n_{m}$; hence $\delta$ may become dependent on $x$ even if it did not before.

g) We claim that Definition 2.1d) is equivalent to the formula

$$
\forall \varepsilon>0 \quad \forall x \in \operatorname{dom}(f) \exists \delta>0: \quad \Phi(f, \varepsilon, x, \delta)
$$

where $\Phi(f, \varepsilon, x, \delta)$ abbreviates the predicate

$\forall x^{\prime} \in B(x, \delta) \cap \operatorname{dom}(f) \exists y^{\prime} \in f\left(x^{\prime}\right) \forall x^{\prime \prime} \in B(x, \delta) \cap \operatorname{dom}(f) \exists y^{\prime \prime} \in f\left(x^{\prime \prime}\right): e\left(y^{\prime}, y^{\prime \prime}\right)<\varepsilon$

Indeed, $x^{\prime}, x^{\prime \prime} \in B(x, \delta)$ yield $y^{\prime} \in f\left(x^{\prime}\right) \cap B(y, \varepsilon)$ and $y^{\prime \prime} \in f\left(x^{\prime \prime}\right) \cap B(y, \varepsilon)$, hence $e\left(y^{\prime}, y^{\prime \prime}\right)<2 \varepsilon$ by the triangle inequality; and, conversely, $x^{\prime}:=x$ yields $y \in f(x)$. Next observe that, again by the triangle inequality, $\Phi(f, \varepsilon, x, \delta)$ implies $\Phi(f, \varepsilon, z, \delta / 2)$ for all $z \in B(x, \delta / 2) \cap \operatorname{dom}(f)$. Now for arbitrary but fixed $\varepsilon$ and to every $x \in \operatorname{dom}(f)$ there exists by hypothesis some $0<\delta=\delta(x)$ 
such that $\Phi(f, \varepsilon, x, \delta(x))$ holds. The open sets $B(x, \delta(x) / 2)$ cover $\operatorname{dom}(f)$; and by compactness, finitely many of them suffice to do so: say, $B\left(x, \delta\left(x_{i}\right) / 2\right)$, $i=1, \ldots, I$. Now take $\bar{\delta}>0$ as the minimum over these finitely many $\delta\left(x_{i}\right) / 2$ : it will satisfy $\Phi(f, \varepsilon, x, \bar{\delta})$ for all $x \in \operatorname{dom}(f)$. Again choosing $x^{\prime}:=x$ in $\Phi$ shows that $f$ is semi-uniformly weakly continuous.

h) Similarly to g), consider the predicate

$$
\begin{aligned}
\forall \varepsilon>0 \quad \forall x \in \operatorname{dom}(f) \quad \forall y \in f(x) \quad \exists \delta \in(0, \varepsilon) \\
\quad \underbrace{\forall x^{\prime}, x^{\prime \prime} \in B(x, \delta) \cap \operatorname{dom}(f) \quad \forall y^{\prime} \in f\left(x^{\prime}\right) \cap B(y, \delta) \quad \exists y^{\prime \prime} \in f\left(x^{\prime \prime}\right) \cap B\left(y^{\prime}, \varepsilon\right)}_{=: \Phi(f, \varepsilon, x, y, \delta)}
\end{aligned}
$$

and note that it is equivalent to strong continuity: The restriction to $\delta<\varepsilon$ is no loss of generality; $y^{\prime} \in B(y, \delta)$ and $y^{\prime \prime} \in f\left(x^{\prime \prime}\right) \cap B(y, \varepsilon)$ according to a) implies $e\left(y^{\prime}, y^{\prime \prime}\right)<\delta+\varepsilon<2 \varepsilon$ arbitrary; whereas, conversely, strong continuity is recovered with $x^{\prime}:=x$ and $y^{\prime}:=y$. Finally, $\Phi(f, \varepsilon, x, y, \delta)$ implies $\Phi(f, \varepsilon, x, \bar{y}, \delta / 2)$ for all $\bar{y} \in B(y, \delta / 2)$. Now fixing $\varepsilon$ and $x$, by hypothesis there exists to each $y \in f(x)$ some $\delta=\delta(y)$ satisfying $\Phi(f, \varepsilon, x, y, \delta(y))$. The balls $B(y, \delta(y) / 2), y \in f(x)$, cover $f(x)$; and by compactness, finitely many of them suffice to do so. Take $\bar{\delta}$ as their minimum, independent of $y$. Then $\Phi(f, \varepsilon, x, y, \bar{\delta})$ holds for all $y \in f(x)$ : uniform strong continuity of $f$.

j) This time abbreviate

$$
\Phi(f, x, y, \varepsilon, \delta) \quad:=\quad \forall x^{\prime} \in B(x, \delta) \cap \operatorname{dom}(f) \quad \exists y^{\prime} \in f\left(x^{\prime}\right) \cap B(y, \varepsilon)
$$

and observe that strong continuity $\forall \varepsilon>0 \forall(x, y) \in f \exists \delta>0 \quad \Phi(f, x, y, \varepsilon / 2, \delta)$ is equivalent to $\forall \varepsilon>0 \forall(x, y) \in f \exists \delta>0 \quad \Phi(f, x, y, \varepsilon, \delta / 2)$. Moreover, $\Phi(f, x, y, \varepsilon / 2, \delta)$ and $(\bar{x}, \bar{y}) \in f \cap(B(x, \delta / 2) \times B(y, \varepsilon / 2))$ together imply $\Phi(f, \bar{x}, \bar{y}, \varepsilon, \delta / 2)$. For fixed $\varepsilon>0$ there exists by hypothesis to each $(x, y) \in f$ some $\delta=\delta(x, y)$ such that $\Phi(f, x, y, \varepsilon / 2, \delta)$. The open balls $B(x, \delta(x, y) / 2) \times B(y, \varepsilon / 2),(x, y) \in f$, thus cover $f$; and by compactness, already finitely many of them suffice to do so. Taking $\bar{\delta}$ as the minimum of their corresponding $\delta(x, y)$, we conclude that $\Phi(f, x, y, \varepsilon, \bar{\delta} / 2)$ holds for all $(x, y) \in f$ : uniform strong continuity.

k) Let $U_{i} \subseteq X(i \in I)$ denote an open covering of $\operatorname{dom}(f)$. Then $U_{i} \times Y$ is an open covering of $f$, hence contains a finite subcover: whose projection onto the first component is a finite subcover of $U_{i}$.

Similarly, let $V_{j} \subseteq Y(j \in J)$ denote an open covering of $f[S] \subseteq Y$. Then $X \times V_{j}$, together with $(X \backslash S) \times Y$, constitutes an open covering of $f$; hence contains a finite subcover: and the corresponding $V_{j}$ yield a finite subcover of $f[S]$.

Finally, $S:=\{x\}$ is closed and thus also $f[S]=f(x)$. 
$\ell)$ Let $\left(x_{n}, y_{n}\right) \subseteq f$ be a sequence. Since $\left(x_{n}\right) \subseteq X$ compact, it has a converging subsequence; w.l.o.g. $\left(x_{n}\right)$ itself. Now by continuity and single-valuedness, $y_{n}=f\left(x_{n}\right) \rightarrow f(x)$ converges. Thus, $f$ is compact; and homeomorphic to $f^{-1}$.

We say that $f$ is pointwise compact if $f(x) \subseteq Y$ is compact for every $x \in \operatorname{dom}(f)$. Any single-valued $f$ automatically satisfies this condition; which in turn implies being pointwise closed as required in Fact 1.1. Pointwise compactness is essential for semi-uniform weak continuity to imply weak continuity in Lemma 2.2e):

Example 2.3 a) The multifunction from [32, EXAMPLE 27c], namely

$$
f:[-1 ; 1] \rightrightarrows[0 ; 1], \quad 0 \geq x \mapsto[0 ; 1), \quad 0<x \mapsto\{1\}
$$

depicted in Figure 2a), is semi-uniformly weakly continuous but not weakly continuous.

b) The multifunction $g:[0 ; 1] \rightrightarrows[0 ; 1]$ with $\operatorname{graph}(g)=([0 ; 2 / 3) \times\{0\}) \cup$ $((1 / 3 ; 1] \times\{1\})$ depicted in Figure $2 b)$ has compact $\operatorname{dom}(g)$ and $g(x)$ for every $x$ but $\operatorname{graph}(g)$ is not compact. Moreover, $g$ is hemi-uniformly strongly continuous but not uniformly strongly continuous.

c) The relation $(\mathbb{Q} \times(\mathbb{R} \backslash \mathbb{Q})) \cup((\mathbb{R} \backslash \mathbb{Q}) \times \mathbb{Q})$ from [7, EXAMPLE 7.2] is uniformly strongly continuous.

d) Inspired by [7, PROPOSITION 2.3], the relation $g:[-1 ; 1] \rightrightarrows[-1 ; 1]$ depicted in Figure 2c) with graph

$$
\{(x, 0): x \leq 0\} \cup\{(x,-1): x \geq 0\} \cup\left\{\left(x, \frac{1+(-1)^{n}}{n+1}\right): n \in \mathbb{N}, \frac{1}{n+1} \leq x \leq \frac{1}{n}\right\}
$$

is compact and both weakly continuous and semi-uniformly weakly continuous but not strongly continuous.

Proof a) To assert semi-uniform weak continuity, consider $\delta=\delta(\varepsilon):=\varepsilon$. Moreover let $y=y(x, \varepsilon):=1$ for $x>0$ and $y(x, \varepsilon):=1-\varepsilon / 2$ for $x \leq 0$. Then, in case $x^{\prime}>0$, choose $y^{\prime}:=1$; and in case $x^{\prime} \leq 0$, chose $y^{\prime}:=1-\varepsilon / 2$.

Suppose $f$ is weakly continuous at $x:=0$, i.e. there exists some appropriate $y \in f(x)=[0 ; 1)$. The consider $\varepsilon:=1-y$ and the induced $\delta>0$ as well as $x^{\prime}:=\delta / 2$ : No $y^{\prime} \in f\left(x^{\prime}\right)=\{1\}$ can satisfy $\varepsilon>\left|y^{\prime}-y\right|=1-y$, contradiction.

b) Note $\operatorname{dom}(g)=[0 ; 1]$ and $g(x)=\{0\}$ for $x \leq 1 / 3, g(x)=\{0,1\}$ for $1 / 3<x<2 / 3$, and $g(x)=\{1\}$ for $x \geq 2 / 3$ : all compact. Concerning hemi-uniform strong continuity, for $x \leq 1 / 3$ let $\delta:=1 / 3$ and $y^{\prime}:=0=y$; for 
$x \geq 2 / 3$ let $\delta:=1 / 3$ and $y^{\prime}:=1=y$; whereas for $1 / 3<x<2 / 3$, choose $\delta:=\min (2 / 3-x, x-1 / 3)$ and $y^{\prime}:=y$. Uniform strong continuity leads to a contradiction when considering $x:=1 / 3+\delta / 2$ and $y:=1$ and $x^{\prime}:=1 / 3$.

c) Let $\delta:=1$; then observe that $\mathbb{Q}$ is dense in $\mathbb{R} \backslash \mathbb{Q}$ and $\mathbb{R} \backslash \mathbb{Q}$ is dense in $\mathbb{Q}$.

d) Let $\tilde{g}(x):=x$ for $x \leq 0$ and $\tilde{g}(x):=\frac{1+(-1)^{n}}{n+1}$ for $\frac{1}{n+1}<x \leq \frac{1}{n}$. Then $\tilde{g}$ tightens $g$ and satisfies, for $x, x^{\prime} \geq 0,0 \leq \tilde{g}(x) \leq 2 x$ as well as $\left|\tilde{g}(x)-\tilde{g}\left(x^{\prime}\right)\right| \leq 2 \max \left(x, x^{\prime}\right)$. Now concerning weak continuity of $g$, in case $x \leq 0$ choose $y:=0$ and $\delta:=\varepsilon / 2$ : then, to $x^{\prime} \in B(x, \delta), y^{\prime}:=\tilde{g}\left(x^{\prime}\right)$ will do. In case $x>0$ choose $y:=-1$ and $\delta:=x$ since $x^{\prime} \in B(x, \delta)$ implies $x^{\prime}>0$ and $y^{\prime}:=-1$ works.

Regarding semi-uniform weak continuity, let $\delta:=\varepsilon / 4$ and distinguish cases $x<\varepsilon / 4$ and $x \geq \varepsilon / 4$. In the former case, $y:=\tilde{g}(x)$ and $y^{\prime}:=\tilde{g}\left(x^{\prime}\right)$ imply $\left|y-y^{\prime}\right| \leq 2 \max \left(x, x^{\prime}\right)<\varepsilon$ for $\left|x-x^{\prime}\right|<\delta$; in the latter case, $y:=-1=: y^{\prime}$ works.

Strong continuity is violated, e.g., at $(x, y)=(1 / 2,2 / 3)$ for $\varepsilon:=1 / 4$.

\subsection{Continuity and Computability of Relations}

Recall that (relative) computability of a multifunction $f: \subseteq \mathbb{R} \rightrightarrows \mathbb{R}$ means that some (oracle) Turing machine can, upon input of any sequence of integer fractions $a_{n} / b_{n}$ with $\left|x-a_{n} / b_{n}\right| \leq 2^{-n}$ for every $n \in \mathbb{N}$ and some $x \in \operatorname{dom}(f)$, output a sequence $u_{m} / v_{m}$ of integer fractions with $\left|y-u_{m} / v_{m}\right| \leq 2^{-m}$ for every $m \in \mathbb{N}$ and some $y \in f(x)$. More generally, a multifunction $f: \subseteq A \rightrightarrows B$ between represented spaces $(A, \alpha)$ and $(B, \beta)$ is considered (relatively) computable if it admits a (relatively) computable $(\alpha, \beta)$-realizer, that is a function $F: \subseteq \Sigma^{\omega} \rightarrow \Sigma^{\omega}$ mapping every $\alpha$-name of some $a \in \operatorname{dom}(f)$ to a $\beta$-name of some $b \in f(a)$ [28, DEFINITION 3.1.3].

Lemma 2.4 Define composition of multifunction $f: \subseteq X \rightrightarrows Y$ and $g: \subseteq Y \rightrightarrows Z$ as

(1) $g \circ f:=\{(x, z) \mid x \in X, z \in Z, f(x) \subseteq \operatorname{dom}(g), \exists y \in Y:(x, y) \in f \wedge(y, z) \in g\}$.

a) $\operatorname{id}_{X}$ tightens $f^{-1} \circ f$; if $f$ is single-valued, then $f \circ f^{-1}=\operatorname{id}_{\text {range }(f)}$.

b) If $f^{\prime}$ tightens $f$ and $g^{\prime}$ tightens $g$, then $g^{\prime} \circ f^{\prime}$ tightens $g \circ f$.

c) If range $(f) \subseteq \operatorname{dom}(g)$ holds and both $f$ and $g$ are compact, then so is $g \circ f$.

d) If $\operatorname{range}(f) \subseteq \operatorname{dom}(g)$ holds and if both $f$ and $g$ map compact sets to compact sets, then so does $g \circ f$.

e) Let $\alpha$ be the representation of $X$, and $\beta$ of $Y$. A multifunction $F: \subseteq \Sigma^{\omega} \rightrightarrows \Sigma^{\omega}$ tightens $\beta^{-1} \circ f \circ \alpha$ iff $\beta \circ F \circ \alpha^{-1}$ tightens $f$. 
f) A function $F: \subseteq \Sigma^{\omega} \rightarrow \Sigma^{\omega}$ is an $(\alpha, \beta)$-realizer of $f$ iff $F$ tightens $\beta^{-1} \circ f \circ \alpha$ iff $\beta \circ F \circ \alpha^{-1}$ tightens $f$.

g) If $F$ realizes $f$ and $G$ realizes $g$, then $G \circ F$ realizes $g \circ f$.

Claim b) appears in WeinRAuCh's [30, Lemma 8.3]. Motivated by f), let us call a multifunction $F$ as in e) an $(\alpha, \beta)$-multirealizer of $f$.

Proof a) Note $f(x) \subseteq \operatorname{dom}\left(f^{-1}\right)$ and $f^{-1} \circ f=\left\{\left(x, x^{\prime}\right): \exists y:(x, y),\left(x^{\prime}, y\right) \in f\right\}$.

b) Note $\operatorname{dom}(g \circ f)=\{x \in \operatorname{dom}(f): f(x) \subseteq \operatorname{dom}(g)\}$; hence $\operatorname{dom}(f) \subseteq \operatorname{dom}\left(f^{\prime}\right) \wedge$ $\operatorname{dom}(g) \subseteq \operatorname{dom}\left(g^{\prime}\right) \wedge f(x) \supseteq f^{\prime}(x) \wedge g(y) \supseteq g^{\prime}(y)$ implies $\operatorname{dom}(g \circ f) \subseteq \operatorname{dom}\left(g^{\prime} \circ f^{\prime}\right)$ as well as $\left(g^{\prime} \circ f^{\prime}\right)(x)=\left\{z: \exists y \in f^{\prime}(x): z \in g^{\prime}(y)\right\} \subseteq(g \circ f)(x)$.

c) Since range $(f) \subseteq \operatorname{dom}(g), g \circ f$ is the image of compact $(f \times \operatorname{range}(g)) \cap$ $(\operatorname{dom}(f) \times g) \subseteq X \times Y \times Z$ under the continuous projection $\Pi_{1,3}: X \times Y \times Z \ni$ $(x, y, z) \mapsto(x, z) \in X \times Z$.

d) immediate from $(g \circ f)[S]=g[f[S]]$, holding under the hypothesis range $(f) \subseteq$ $\operatorname{dom}(g)$.

e) If $F$ tightens $\beta^{-1} \circ f \circ \alpha$, then $\beta \circ F \circ \alpha^{-1}$ tightens $\beta \circ \beta^{-1} \circ f \circ \alpha \circ \alpha^{-1}$ due to $b$ ); which in turn coincides with $\operatorname{id}_{X} \circ f \circ \operatorname{id}_{Y}=f$ according to a).

Conversely, $F=\operatorname{id}_{\Sigma^{\omega}} \circ F \circ \operatorname{id}_{\Sigma^{\omega}}$ tightens $\beta^{-1} \circ \beta \circ F \circ \alpha^{-1} \circ \alpha$ by hypothesis, a) and b).

f) $F$ being an $(\alpha, \beta)$-realizer of $f$ means $\operatorname{dom}(F) \supseteq \operatorname{dom}(f \circ \alpha)$ and $\beta(F(\bar{\sigma})) \in$ $f(\alpha(\bar{\sigma}))$ for every $\bar{\sigma} \in \operatorname{dom}(f \circ \alpha)=\operatorname{dom}\left(\beta^{-1} \circ f \circ \alpha\right)$; now apply e).

g) Straightforward.

The above notion of composition for relations is, like that of 'tightening', from [30, SECTION 3]. Note that property g) would fail, if the requirement $f(x) \subseteq \operatorname{dom}(g)$ were dropped from the definition of $g \circ f$. Mapping compact sets to compact sets is a property which turns out useful below. It includes both compact relations (Lemma 2.2k) and continuous functions:

Example 2.5 a) Let $f: X \rightarrow Y$ be a single-valued continuous function. Then $f$ maps compact sets to compact sets.

b) The inverse $\left(\rho_{\mathrm{sd}}^{d}\right)^{-1}$ of the $d$-dimensional signed digit representation maps compact set to compact sets.

c) The functions id : $x \rightarrow x$ and sgn : $\mathbb{R} \rightarrow\{-1,0,1\}$ both map compact sets to compact sets; however their Cartesian product id $\times$ sgn does not map compact $\{(x, x):-1 \leq x \leq 1\}$ to a compact set. 
Indeed, the signed digit representation $\rho_{\text {sd }}$ is well-known proper [28, pp.209-210], i.e. preimages of compact sets are compact.

Focusing on complete separable metric spaces and pointwise compact multifunctions, strong continuity is in view of Fact 1.1 (in general strictly) stronger than relative computability; whereas weak continuity is (again in general strictly) weaker than relative computability:

Example 2.6 a) The relation from Example 2.3d) is not computable relative to any oracle.

b) The relation from Example 2.3c) is (uniformly strongly continuous but, lacking pointwise compactness) not computable relative to any oracle.

c) The closure of the relation from Example 2.3b), that is with graph $([0 ; 2 / 3] \times$ $\{0\}) \cup([1 / 3 ; 1] \times\{1\})$, is computable but not strongly continuous.

Proof a) by contradiction: Suppose some oracle machine $\mathcal{M}$ computes this relation. On input of the rational sequence $(0,0,0, \ldots)$ as a $\rho$-name of $x:=0$ it thus outputs a $\rho$-name of $y=0$, i.e. a rational sequence $\left(p_{m}\right)$ with $\left|p_{m}\right|<2^{-m}$. In particular it prints $p_{1}>-1 / 2$ after having read only finitely many elements from the input sequence; say, up to the $(N-1)$-st element. Now consider the behavior of $\mathcal{M}$ on the input sequence $\left(0,0, \ldots, 0,2^{-N}, 2^{-N}, \ldots\right)$ as $\rho$-name of $x^{\prime}:=2^{-N}$ : Its output sequence $\left(p_{m}^{\prime}\right)$ will, again, begin with $p_{1}^{\prime}=p_{1}>-1 / 2$ and thus cannot be a $\rho$-name of -1 . Since $g\left(x^{\prime}\right)=\left\{-1,0,2 /\left(1+2^{N}\right)\right\}$, it must therefore satisfy $\left|p_{m}^{\prime}-y\right|<2^{-m}$ for all $m$ and for one of $y=0=: y_{0}$ or $y=2 /\left(1+2^{N}\right)=: y_{1}$. In particular, $p_{N+1}^{\prime}$ satisfies $y_{j} \in B\left(p_{N+1}^{\prime}, 2^{-N-1}\right) \not \supset y_{1-j}$ for the unique $j \in\{0,1\}$ with $y=y_{j}$ and is printed upon reading only the first, say, $N^{\prime} \geq N$ elements of $\left(0,0, \ldots, 2^{-N}, 2^{-N}, \ldots\right)$. Finally it is easy to extend this finite sequence to a $\rho$-name of some $x^{\prime \prime}$ close to $x^{\prime}$ with $y_{j} \notin g\left(x^{\prime \prime}\right) \ni y_{1-j}$; and upon this input $\mathcal{M}$ will now, again, output elements $p_{1}^{\prime}, \ldots, p_{N+1}^{\prime}$ which, however, cannot be extended to a $\rho$-name of any $y^{\prime \prime} \in g\left(x^{\prime \prime}\right)$ : contradiction.

b) see $[7$, p.24].

c) Immediate.

For relations with discrete range, on the other hand, weak continuity does characterize relative computability:

Theorem 2.7 Let $(X, d, A, \alpha)$ and $(Y, e, B, \beta)$ be computable metric spaces according to [28, DEFINITION 8.1.2].

If $Y$ is discrete and $f: \subseteq X \rightrightarrows Y$ is weakly continuous, then $f$ is relatively computable. 
Proof Since $(Y, e)$ is discrete, to every $y \in Y$ there exists some $\varepsilon=\varepsilon(y)>0$ such that $Y \cap B(y, \varepsilon(y))=\{y\}$. In particular $Y=B$ is at most countable. Now to $y \in B$ consider the set

$$
U_{y}:=\left\{x \in \operatorname{dom}(f): \exists \delta>0 \forall x^{\prime} \in B(x, \delta) \cap \operatorname{dom}(f) \exists y^{\prime} \in f\left(x^{\prime}\right) \cap B(y, \varepsilon(y))\right\}
$$

and note that it is open in $\operatorname{dom}(f)$ because $y^{\prime} \in B(y, \varepsilon(y))$ requires $y^{\prime}=y$. Hence $U_{y}=\operatorname{dom}(f) \cap \bigcup_{j \in \mathbb{N}} B\left(q_{j, y}, 1 / n_{j, y}\right)$ for certain $n_{j, y} \in \mathbb{N}$ and $q_{j, y} \in A$. Now encode ( $\alpha$-names of) all these $q_{j, y}$ and $n_{j, y}$ as one oracle. Then, given $x \in \operatorname{dom}(f)$, search for some $(j, y)$ with $x \in B\left(q_{j, y}, 1 / n_{j, y}\right) \subseteq U_{y}$ : when found, such $y$ by construction belongs to $f(x)$; and, conversely, weak continuity asserts $x$ to belong to $U_{y}$ for some $y$.

\subsection{Motivation for Uniform Continuity}

Many proofs of uncomputability of relations or of topological lower bounds [32] apply weak continuity as a necessary condition: merely necessary, in view of the above example, and thus of limited applicability. The rest of this work thus explores topological conditions stronger than weak continuity yet necessary for relative computability.

Uniform continuity of functions is such a stronger notion - and an important concept of its own in mathematical analysis - yet does not straightforwardly (or at least not unanimously) extend to multifunctions. Guided by the equivalence between uniform continuity and relative computability for functions with compact graph, our aim is a topological characterization of oracle-computable compact real relations. One such characterization is Fact 1.1; however we would like to avoid (second-order) quantifying over tightenings.

To this end observe that every (relatively) computable function $f$ is (relatively) effectively locally uniformly continuous [28, THEOREM 6.2.7], that is, uniformly continuous on every compact subset $K \subseteq \operatorname{dom}(f)$ [16]:

$$
\forall \varepsilon>0 \exists \delta>0 \forall x \in K \forall x^{\prime} \in B(x, \delta) \cap K: d\left(f(x), f\left(x^{\prime}\right)\right)<\varepsilon .
$$

This suggests to look for related concepts for multifunctions, i.e. where $\delta$ does not depend on $x$. Semi-uniform weak continuity in the sense of Definition 2.1c), however, fails to strengthen weak continuity because it allows $y$ to depend on $\varepsilon$.

\section{Henkin-Continuity}

In view of the above discussion, we seek for an order on the four quantifiers

$$
\forall x \in \operatorname{dom}(f), \quad \exists y \in f(x), \quad \forall \varepsilon>0, \quad \exists \delta>0
$$


such that $y$ does not depend on $\varepsilon$ and $\delta$ does not depend on $x$. This cannot be expressed in classical first-order logic and has spurred the introduction of the non-classical so-called Henkin Quantifier

$$
\mathrm{Q}_{\mathrm{H}}(x, y, \varepsilon, \delta)=\left(\begin{array}{cc}
\forall x & \exists y \\
\forall \varepsilon & \exists \delta
\end{array}\right)
$$

where the suggestive writing indicates that very condition: that $y$ may depend on $x$ but not on $\varepsilon$ while $\delta$ may depend on $\varepsilon$ but not on $x$; compare BARWISE [1], KRYNICKI and MostowsKi [17], and VÄÄNÄNEN [27]. More formally we consider $\mathrm{Q}_{H}$ equipped with the semantics of existential second-order logic via Skolem functions:

$$
\mathrm{Q}_{\mathrm{H}}(a, b, u, v) \varphi(a, b, u, v) \quad: \Leftrightarrow \quad \exists B, V: \forall a \forall u: \varphi(a, B(a), u, V(u))
$$

Following BEESON [5, p.380], consider the following notion of uniform continuity for a partial relation $f: \subseteq X \rightrightarrows Y$ between metric ${ }^{\mathbb{I}}$ spaces $X$ and $Y$ :

Definition 3.1 Call $f$ Henkin-continuous if the following holds:

(2) $\left(\begin{array}{cc}\forall \varepsilon>0 & \exists \delta>0 \\ \forall x \in \operatorname{dom}(f) & \exists y \in f(x)\end{array}\right) \quad \forall x^{\prime} \in B(x, \delta) \cap \operatorname{dom}(f) \quad \exists y^{\prime} \in B(y, \varepsilon) \cap f\left(x^{\prime}\right)$.

Observe that uniform strong continuity implies Henkin-continuity; from which in turn follows both weak continuity and semi-uniform weak continuity. In fact, Henkincontinuity is strictly stronger than the latter two:

Example 3.2 a) The relation $g$ from Example 2.3d) and Example 2.6a) is (compact and both weakly continuous and semi-uniformly weakly continuous but) not Henkin-continuous.

b) It does, however, satisfy

$$
\left(\begin{array}{l}
\forall \varepsilon>0 \quad \exists \delta>0 \\
\forall x, x^{\prime} \exists y \in g(x)
\end{array}\right) \quad \exists y^{\prime} \in g\left(x^{\prime}\right) \quad\left(x^{\prime} \in B(x, \delta) \Rightarrow y^{\prime} \in B(y, \varepsilon)\right)
$$

c) The relations from Example 2.3b) and Example 2.6c) are (computable and) Henkin-continuous.

Proof a) by contradiction: Suppose $y=y(x)$ satisfies Equation (2). Now let $\varepsilon:=1 / 2$ and consider $\delta:=\delta(\varepsilon)$ according to Equation (2). Then $y(x)=-1$ is impossible for all $0<x<\delta$, as $x^{\prime}:=(x-\delta) / 2<0$ implies $g\left(x^{\prime}\right)=\{0\}$

\footnotetext{
"Its generalization to uniform spaces is immediate but beyond our purpose.
} 
which is disjoint to $B(y, \varepsilon)$. Now consider $\varepsilon^{\prime}:=\delta / 2$ and $\delta^{\prime}:=\delta\left(\varepsilon^{\prime}\right)$. We claim that $y(x)=-1$ is necessary for all $x=1 /(n+1)>\varepsilon^{\prime}, n \in \mathbb{N}$, this leading to a contradiction for $\delta / 2<x<\delta$. Indeed in case $y(x)=2 /(n+1), n$ even, every sufficiently close $x^{\prime}<x$ has $g\left(x^{\prime}\right)=\{0,-1\}$ which is disjoint to $B(y, \varepsilon)$; whereas in case $y(x)=0$, every sufficiently close $x^{\prime}>x$ has $g\left(x^{\prime}\right)=\{2 /(n+1),-1\}$ again disjoint to $B(y, \varepsilon)$.

b) Let $\delta:=\varepsilon$ and take $y:=-1$ in case $x, x^{\prime}>0 ; y:=0$ in case $x \leq 0$; and $\{y\}:=g(x) \cap[0 ; 1]$ in case $x^{\prime} \leq 0<x$.

c) For $x \leq \frac{1}{2}$ choose $y:=0$ and for $x>\frac{1}{2}$ choose $y:=1$; independently, choose $\delta:=\frac{1}{6}$.

\subsection{Further Examples and Some Properties}

Remember that, for single-valued functions, Henkin-continuity coincides with uniform continuity. The sequel employs infinite strings over both alphabets $\{0,1\}$ and over $\Sigma^{\prime}=\{0,1, \overline{1},$.$\} , tacitly presuming one is encoded over the other via 0 \mapsto 00,1 \mapsto 10$, $\overline{1} \mapsto 01, . \mapsto 11$. This bijection $\iota: \Sigma^{\prime \omega} \rightarrow\{0,1\}^{\omega}$ satisfies $d\left(\iota\left(\bar{\sigma}^{\prime}\right), \iota\left(\bar{\tau}^{\prime}\right)\right) \leq$ $\left(d^{\prime}\left(\bar{\sigma}^{\prime}, \bar{\tau}^{\prime}\right)\right) \leq 2 d\left(\iota\left(\bar{\sigma}^{\prime}\right), \iota\left(\bar{\tau}^{\prime}\right)\right)$, where $d:(\bar{\sigma}, \bar{\tau}) \mapsto 2^{-\min \left\{k=0,1,2, \ldots ; \sigma_{k} \neq \tau_{k}\right\}}$ and $d^{\prime}:$ $\left(\bar{\sigma}^{\prime}, \bar{\tau}^{\prime}\right) \mapsto 2^{-\min \left\{k=0,1,2, \ldots: \sigma_{k}^{\prime} \neq \tau_{k}^{\prime}\right\}}$ denotes the metrics on $\{0,1\}^{\omega}$ and $\Sigma^{\prime \omega}$, respectively.

Example 3.3 Recall from the Type-2 Theory of Effectivity (TTE) the Cauchy representation $\rho_{\mathrm{C}}$ [28, DEFINITION 4.1.5] and the signed digit representation $\rho_{\mathrm{sd}}[28$, DEFINITION 7.2.4] of real numbers.

a) $\rho_{\mathrm{sd}}: \subseteq \Sigma^{\prime \omega} \rightarrow \mathbb{R}$ is not uniformly continuous

b) nor is the restriction $\left.\rho_{\mathrm{C}}\right|^{[0 ; 1]}: \subseteq \Sigma^{\omega} \rightarrow[0 ; 1]$; compare [28, EXAMPLE 7.2.3].

c) However for every compact $K \subseteq \mathbb{R}$ the restriction $\left.\rho_{\mathrm{sd}}\right|^{K}: \subseteq \Sigma^{\prime \omega} \rightarrow K$ is uniformly (i.e. Henkin-) continuous. More precisely, if $L \in \mathbb{N}$ is a bound on $K$, then $\left.\rho_{\mathrm{sd}}\right|^{K}$ is (4L)-Lipschitz continuous.

d) and so are the restrictions $\left.\rho_{\mathrm{C}}\right|_{C}: C \rightarrow \mathbb{R}$ and $\left.\rho_{\mathrm{sd}}\right|_{C}: C \rightarrow \mathbb{R}$ for any compact $C \subseteq \Sigma^{\omega}$ with, respectively, $C \subseteq \operatorname{dom}\left(\rho_{\mathrm{C}}\right)$ and $C \subseteq \operatorname{dom}\left(\rho_{\mathrm{sd}}\right)$.

e) $\rho_{\mathrm{C}}^{-1}: \mathbb{R} \rightrightarrows \Sigma^{\omega}, \quad \mathbb{R} \ni x \mapsto\left\{\bar{\sigma}: \rho_{\mathrm{C}}(\bar{\sigma})=x\right\}$, the inverse of the Cauchy representation, is Henkin-continuous.

Proof a) Consider some large integer $x=2^{k} \in \mathbb{N}$ with $\rho_{\text {sd }}$-name $10 \cdots 0.0 \cdots$. Then modifying this name $\bar{\sigma}$ at the $k$-th position affects the value $\rho_{\text {sd }}(\bar{\sigma})$ by an absolute value of 1 . In particular, to $\varepsilon:=1, \delta>0$ satisfying

$$
d(\bar{\sigma}, \bar{\tau})<\delta \Rightarrow d\left(\rho_{\mathrm{sd}}(\bar{\sigma}), \rho_{\mathrm{sd}}(\bar{\tau})\right)<\varepsilon
$$


must depend on the value of $x=2^{k}$, i.e. on $\bar{\sigma}$.

b) Fix $k \in \mathbb{N}$, and consider integers $a_{n}:=2^{k+n}$ and $b_{n}:=3 \cdot 2^{k+n}$. Hence the concatenation $\bar{\sigma}$ of binary-encoded numerators $a_{n}$ and denominators $b_{n}$ constitutes a $\rho_{\mathrm{C}}$-name of $x:=1 / 3$. Note that the secondmost-significant digit of $b_{1}$ resides roughly at position $\# k$ in $\bar{\sigma}$. Hence switching to $a_{n}^{\prime}:=a_{n}$ and $b_{n}^{\prime}:=2 \cdot 2^{k+n}$ yields $\bar{\sigma}^{\prime}$ of metric distance to $\bar{\sigma}$ of order $\delta=2^{-k}$; whereas the value $x^{\prime}=\rho_{\mathrm{C}}\left(\bar{\sigma}^{\prime}\right)=1 / 2$ changes by $\varepsilon=1 / 6$.

c) First consider the case $K=[0 ; 1]$. Then, modifying the $k$-th digit $b_{k} \in\{0,1, \overline{1}\}$ of a signed digit expansion $\sum_{n=0}^{\infty} b_{n} 2^{-n}$ affects its value by no more than $2^{-k}$. In the general case, let $L=2^{\ell}$ denote a bound on $K$. Then, similarly, modifying the $k$-th position of a signed digit expansion $\sum_{n=-N}^{\infty} b_{n} 2^{-n}$ affects its value by no more than $2^{\ell-k}$.

d) Like any admissible representation, $\rho_{\mathrm{C}}$ and $\rho_{\mathrm{sd}}$ are continuous; hence uniformly continuous on compact subsets.

e) To $\varepsilon=2^{-k}>0$ let $\delta:=2^{-k}$. Now consider arbitrary $x \in \mathbb{R}$ and as $\rho_{\mathrm{C}^{-}}$ name $\bar{\sigma}$ the (binary encodings of numerators and denominators of the) dyadic sequence $q_{n}:=\left\lfloor x \cdot 2^{n+1}\right\rfloor / 2^{n+1}$. In fact it holds $\left|x-q_{n}\right| \leq 2^{-n-1} \leq 2^{-n}$. Now $x^{\prime} \in B\left(x^{\prime}, \delta\right)$ has $\left|x^{\prime}-q_{n}\right| \leq 2^{-k}+2^{-n-1} \leq 2^{-n}$ for $n \leq k-1$. Therefore the first $k-1$ elements of $\left(q_{n}\right)$, and in particular the first $k-1$ symbols of $\bar{\sigma}$, extend to a $\rho_{\mathrm{C}}-$ name $\bar{\tau}$ of $x^{\prime}$; i.e. such that $d(\bar{\sigma}, \bar{\tau})<\varepsilon$.

A classical property both of continuity and uniform continuity is closure under restriction and under composition. Also Henkin-continuity passes these (appropriately generalized) sanity checks:

Observation 3.4 a) Let $f: \subseteq X \times Y$ be Henkin-continuous and tighten $g: \subseteq X \times Y$. Then $g$ is Henkin-continuous, too.

b) If $f: \subseteq X \times Y$ and $g: \subseteq Y \times Z$ are Henkin-continuous, then so is $g \circ f \subseteq X \times Z$.

Proof a) For $g$ loosening $f$ and in the definition of Henkin-continuity of $g$, the universal quantifiers range over a subset, and the existential quantifiers range over a superset, of those in the definition of Henkin-continuity of $f$. 
b) By hypothesis we have:

$$
\begin{aligned}
& \text { (3) }\left(\begin{array}{cc}
\forall \varepsilon>0 & \exists \delta>0 \\
\forall y \in \operatorname{dom}(g) & \exists z \in g(y)
\end{array}\right) \quad \forall y^{\prime} \in B(y, \delta) \cap \operatorname{dom}(g) \quad \exists z^{\prime} \in B(z, \varepsilon) \cap g\left(y^{\prime}\right) \\
& \text { (4) }\left(\begin{array}{cc}
\forall \delta>0 & \exists \gamma>0 \\
\forall x \in \operatorname{dom}(f) & \exists y \in f(x)
\end{array}\right) \quad \forall x^{\prime} \in B(x, \gamma) \cap \operatorname{dom}(f) \quad \exists y^{\prime} \in B(y, \delta) \cap f\left(x^{\prime}\right)
\end{aligned}
$$

Thus, to $\varepsilon>0$, take $\delta>0$ according to Equation (3) and in turn $\gamma>0$ according to Equation (4). Similarly, to $x \in \operatorname{dom}(g \circ f) \subseteq \operatorname{dom}(f)$, take $y \in f(x) \subseteq \operatorname{dom}(g)$ according to Equations (4) and (1); and in turn $z \in g(y)$ according to Equation (3). This $z$ thus belongs to $(g \circ f)(x)$ and was obtained independently of $\varepsilon$, nor does $\gamma$ depend on $x$. Moreover to $x^{\prime} \in B(x, \gamma) \cap \operatorname{dom}(g \circ f)$ there is a $y^{\prime} \in B(y, \delta) \cap f\left(x^{\prime}\right) \subseteq B(y, \delta) \cap \operatorname{dom}(g)$; to which in turn there is a $z^{\prime} \in B(z, \varepsilon) \cap g\left(y^{\prime}\right)$, i.e. $z^{\prime} \in B(z, \varepsilon) \cap(g \circ f)\left(x^{\prime}\right)$.

The following further example in Item b) turns out as rather useful:

Proposition $3.5 \quad$ a) Every $x \in \mathbb{R}$ has a signed digit expansion

$$
x=\sum_{n=-N}^{\infty} a_{n} 2^{-n}, \quad a_{n} \in\{0,1, \overline{1}\}
$$

with no consecutive digit pair 11 nor $\overline{1} \overline{1}$ nor $1 \overline{1}$ nor $\overline{1} 1$.

b) For $k \in \mathbb{N}$, each $|x| \leq \frac{2}{3} \cdot 2^{-k}$ admits such an expansion with $a_{n}=0$ for all $n \leq k$. And, conversely, $x=\sum_{n=k+1}^{\infty} a_{n} 2^{-n}$ with $\left(a_{n}, a_{n+1}\right) \in\{10, \overline{1} 0,01,0 \overline{1}, 00\}$ for every $n$ requires $|x| \leq \frac{2}{3} \cdot 2^{-k}$.

c) Let $x=\sum_{n=-N}^{\infty} a_{n} 2^{-n}$ be a signed digit expansion and $k \in \mathbb{N}$ such that $\left(a_{n}, a_{n+1}\right) \in\{10, \overline{1} 0,01,0 \overline{1}, 00\}$ for each $n>k$. Then every $x^{\prime} \in[x-$ $\left.2^{-k} / 3 ; x+2^{-k} / 3\right]$ admits a signed digit expansion $x^{\prime}=\sum_{n=-N}^{\infty} b_{n} 2^{-n}$ with $a_{n}=b_{n} \forall n \leq k$.

d) The inverse $\rho_{\mathrm{sd}}^{-1}: \mathbb{R} \rightrightarrows \Sigma^{\prime \omega}$ of the signed digit representation is Henkincontinuous.

Proof a) Begin with an arbitrary signed digit expansion $\left(a_{n}\right)$ of $x$, preceded by 0 , and replace, starting from the most significant digits,

i) any occurrence of 011 with $10 \overline{1}$,

ii) any occurrence of $0 \overline{1} \overline{1}$ with $\overline{1} 01$,

iii) any occurrence of $01 \overline{1}$ with 001 , 
iv) any occurrence of $0 \overline{1} 1$ with $00 \overline{1}$.

Note that these substitutions do not affect the value $\sum_{n=-N}^{\infty} a_{n} 2^{-n}$. Moreover the above four cases are the only possible involving one of 11 or $\overline{1} \overline{1}$ or $1 \overline{1}$ or $\overline{1} 1$ because, by induction hypothesis and proceeding from left (most significant) to right, no such combination was left before of the current position. On the other hand, rewriting Rule i) may well introduce a new occurrence of 11 or $\overline{1} 1$ before the current position; this is illustrated in the example of 0101011. Similarly for $\overline{1} \overline{1}$ or $1 \overline{1}$ in Rule ii). Therefore, we apply the rules in two nested loops:

- An infinite outer one for $n=-N, \ldots, 0,1,2, \ldots$, with $a_{-N}=0$ initially $\|_{\text {, }}$ maintaining that neither 11 nor $\overline{1} \overline{1}$ nor $1 \overline{1}$ nor $\overline{1} 1$ occur before position $n$

- one application of rules i-iv) to remove a possible occurrence at position $n$

- followed by a finite inner loop for $j=n$ downto $-N$ to iteratively remove occurrences which may have been newly introduced at position $j$.

For each $n \geq-N$ let $\bar{a}^{(n)}$ denote the expansion obtained after termination of the corresponding inner loop, that is, satisfying the restriction on adjacent symbol pairs up to position $n$. Now $\left(\bar{a}^{(n)}\right)_{n \in \mathbb{N}}$ need not converge** but, living in a compact space, has accumulation points, which do have the desired properties.

b) Shifting/scaling reduces to the case $k=0$; and negation to the case $x>0$. Now $\frac{2}{3}=0.1010 \ldots$ is an expansion with the claimed properties. However $\frac{2}{3}=1.0 \overline{1} 0 \overline{1} \ldots$ is obviously the smallest real admitting a signed digit expansion that both starts with 1 and no consecutive $11, \overline{1} \overline{1}, 1 \overline{1}, \overline{1} 1$. Thus, for every $0 \leq x<\frac{2}{3}$, the signed digit expansion provided by a) has the claimed properties. For the converse, similarly observe that $0.1010 \ldots$ has the largest value among all signed digit expansions with the claimed properties; and its value is $\frac{2}{3}$.

c) Let $x^{\prime \prime}:=\sum_{n=-N}^{k} a_{n} 2^{-n}$ and observe that $x-x^{\prime \prime}=\sum_{n=k+1}^{\infty} a_{n} 2^{-n}$ is by hypothesis a signed digit expansion satisfying $\left(a_{n}, a_{n+1}\right) \in\{10, \overline{1} 0,01,0 \overline{1}, 00\}$ for all $n \geq k+1$, hence $\left|x-x^{\prime \prime}\right| \leq \frac{2}{3} \cdot 2^{-k}$ by b). In addition with the hypothesis $\left|x-x^{\prime}\right| \leq 2^{-k} / 3$, we conclude that $x^{\prime}-x^{\prime \prime} \in\left[-2^{-k} ; 2^{-k}\right]$ admits an expansion (possibly using combinations like $\overline{1} \overline{1}$ or 11 ) starting only at the $(k+1)$-st digit: $x^{\prime}-x^{\prime \prime}=\sum_{n=k+1}^{\infty} b_{n} 2^{-n}$. Thus $x^{\prime}=\left(x^{\prime}-x^{\prime \prime}\right)+x^{\prime \prime}=$ $\sum_{n=-N}^{k} a_{n} 2^{-n}+\sum_{n=k+1}^{\infty} b_{n} 2^{-n}$ is an expansion with the claimed properties.

d) To $\varepsilon>0$ let $\delta:=\varepsilon / 3$ and $k:=\left\lceil\log _{2} \frac{1}{\varepsilon}\right\rceil$, so that $2^{-k} \leq \varepsilon$ and $\delta \leq 2^{-(k-1)} / 3$. To $x \in \mathbb{R}$ let $\bar{\sigma} \in \Sigma^{\prime \omega}$ be a $\rho_{\text {sd }}$-name encoding a signed digit expansion $\left(a_{n}\right)$

\footnotetext{
"The leading 0 may be changed into 1 or $\overline{1}$, requiring to increment $N$. But this can happen at most once since any signed-digit expansion $\left(a_{n}\right)$ of $|x| \leq 2^{N-1}$ must have $a_{-N-1}=0$.

${ }^{* *}$ The following counterexample is due to VASSILIS GREGORIADES: $0 \overline{1} .011 \overline{1}(1 \overline{1})^{\mathbb{N}} \ldots$
} 
of $x$ according to a). Due to c), every $x^{\prime} \in \bar{B}(x, \delta) \subseteq \bar{B}\left(x, \cdot 2^{-(k-1)} / 3\right)$ admits a signed digit expansion $\left(b_{n}\right)$ coinciding with $\left(a_{n}\right)$ for all $n \leq k-1$. Its encoding $\bar{\tau} \in \Sigma^{\prime \omega}$ thus has $d(\bar{\sigma}, \bar{\tau}) \leq 2^{-k} \leq \varepsilon$.

\subsection{Other Characterizations and Tools}

Let us call a mapping $\lambda: \mathbb{N} \rightarrow \mathbb{N}$ a modulus; and say that a multifunction $f: \subseteq X \rightrightarrows Y$ is $\lambda$-continuous in $(x, y) \in f$ if, to every $m \in \mathbb{N}$ and every $x^{\prime} \in \operatorname{dom}(f) \cap \bar{B}\left(x, 2^{-\lambda(m)}\right)$ there exists some $y^{\prime} \in f\left(x^{\prime}\right) \cap \bar{B}\left(y, 2^{-m}\right)$. Here, $\bar{B}(x, r):=\left\{x^{\prime} \in X: d\left(x, x^{\prime}\right) \leq r\right\}$ denotes the closed ball of radius $r$ around $x$. Now skolemize " $\forall \varepsilon>0 \exists \delta>0$ ":

Observation 3.6 A multifunction $f: \subseteq X \rightrightarrows Y$ is Henkin-continuous iff there exists a modulus $\lambda$ such that, for every $x \in \operatorname{dom}(f)$, there exists $y \in f(x)$ such that $f$ is $\lambda$-continuous in $(x, y)$; equivalently: if, for every $x \in \operatorname{dom}(f), f$ admits some singlevalued total selection $f_{x}: X \rightarrow Y \lambda$-continuous in $\left(x, f_{x}(x)\right)$ (but possibly discontinuous elsewhere, see Example 3.8 below).

Definition 3.7 a) A multifunction $f: \subseteq X \rightrightarrows Y$ is weakly $L$-Lipschitz $(L>0)$ if

$$
\forall x \in \operatorname{dom}(f) \exists y \in f(x) \forall x^{\prime} \in \operatorname{dom}(f) \exists y^{\prime} \in B\left(y, L \cdot d\left(x, x^{\prime}\right)\right) \cap f\left(x^{\prime}\right) .
$$

b) Call a family $f_{i}: \subseteq X_{i} \rightrightarrows Y_{i}(i \in I)$ of multifunctions Henkin-equicontinuous if the following holds:

(6) $\left(\begin{array}{c}\forall \varepsilon>0 \quad \exists \delta>0 \\ \forall i \in I \forall x \in \operatorname{dom}\left(f_{i}\right) \exists y \in f_{i}(x)\end{array}\right) \forall x^{\prime} \in B(x, \delta) \quad \exists y^{\prime} \in B(y, \varepsilon) \cap f_{i}\left(x^{\prime}\right)$.

So every weakly Lipschitz relation is Henkin-continuous; and every family of total weakly $L$-Lipschitz relations is Henkin-equicontinuous. The proof of Proposition 3.5d) provides Item a) of the following

Example 3.8 a) The inverse $\rho_{\mathrm{sd}}^{-1}: \mathbb{R} \rightrightarrows \Sigma^{\prime \omega}$ of the signed digit representation is weakly 3-Lipschitz.

b) The relation

$$
f:=\{(0,0)\} \cup \bigcup_{k \in \mathbb{N}}\left[2^{-k} ; 3 \cdot 2^{-k}\right] \times\left\{2^{-k}\right\} \subseteq[0 ; 3 / 2] \times[0 ; 1]
$$

depicted in Figure 3 is compact and weakly 2-Lipschitz. Moreover, $f$ is computable but has no locally continuous selection in $x_{0}=0$. 


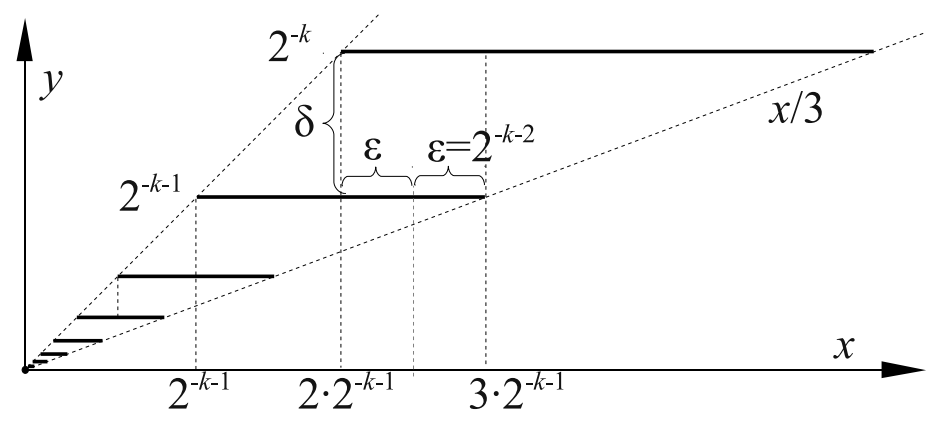

Figure 3: Computable compact relation with no locally continuous selection in $x_{0}=0$.

Proof of Example 3.8b) Figure 3 reveals that the Lipschitz constant 2 is attained at the worst case $x=\frac{5}{2} \cdot 2^{-k-1}$ : For $y=2^{-k}, x^{\prime}<\frac{4}{2} \cdot 2^{-k-1}$ requires $y^{\prime}=2^{-k-1}$; and for $y=2^{-k-1}, x^{\prime}>\frac{6}{2} \cdot 2^{-k-1}$ requires $y^{\prime}=2^{-k}$. On the other hand every $(x, y) \in f$ satisfies $x / 3 \leq y \leq x$; thus the following algorithm computes $f$ : Given $x \in[0 ; 1]$ in form of a nested sequence $\left[a_{n} ; b_{n}\right]$ of intervals with rational endpoints $b_{n}-a_{n} \leq 2^{-n-1}$, test whether $\left[a_{n} ; b_{n}\right] \subseteq\left[2^{-n} ; 3 \cdot 2^{-n}\right]$ holds: if not, output $\left[a_{n} / 3 ; b_{n}\right]$ and proceed to interval $\# n+1$, otherwise switch to outputting the constant sequence $\left[2^{-n} ; 2^{-n}\right]$. Note that for $x=0$, the output sequence $\left[a_{n} / 3 ; b_{n}\right]$ will indeed converge to $y=0$. In case $3 \cdot 2^{-k-1}<x \leq 2 \cdot 2^{-k}$ on the other hand, $\left[a_{k} ; b_{k}\right] \subseteq\left[2^{-k} ; 3 \cdot 2^{-k}\right]$ holds and will result in the output of $y=2^{-k} \in f(x)$, compliant with possible previous intervals $\left[a_{n} / 3 ; b_{n}\right] \supseteq[x / 3 ; x] \supseteq f(x)$. In the final case $2 \cdot 2^{-k-1}<x \leq 3 \cdot 2^{-k-1}$, at least one of $\left[a_{k} ; b_{k}\right] \subseteq\left[2^{-k} ; 3 \cdot 2^{-k}\right]$ and $\left[a_{k} ; b_{k}\right] \subseteq\left[2^{-k-1} ; 3 \cdot 2^{-k-1}\right]$ holds; hence the algorithm will produce $2^{-n}$ either for $n=k$ or for $n=k+1$.

Proposition 3.9 a) Let $I$ denote an ordinal and $f_{i}: \subseteq X \rightrightarrows Y(i \in I)$ an Henkinequicontinuous family of pointwise compact multifunctions that are decreasing in the sense that $f_{j}$ tightens $f_{i}$ whenever $j>i$. Then $f(x):=\bigcap_{i: f_{i}(x) \neq \emptyset} f_{i}(x)$ is again a pointwise compact and Henkin-continuous tightening of each $f_{i}$. Moreover, if all $f_{i}$ are $\lambda$-continuous, then so is $f$.

b) Let $f: X \rightrightarrows Y$ be $\lambda$-continuous and pointwise compact for some modulus $\lambda$. Then $f$ has a minimal $\lambda$-continuous pointwise compact tightening.

c) There is however a decreasing family of Henkin-continuous compact multifunctions whose intersection is not Henkin-continuous.

Proof a) Since the case of a finite $I$ is trivial, it suffices to treat the case $I=\mathbb{N}$ of a sequence; the general case then follows by transfinite induction. Let $x \in \operatorname{dom}\left(f_{i}\right)$. 
Then $f_{j}(x) \subseteq f_{i}(x)$ for each $j>i$, and hence $f(x)=\bigcap_{j \geq i} f_{j}(x) \subseteq f_{i}(x)$ is (compact and) the intersection of non-empty compact decreasing sets: $f(x) \neq \emptyset, x \in$ $\operatorname{dom}(f)$. Moreover let $\varepsilon>0$ be arbitrary and consider an appropriate $\delta$ according to Equation (6) independent of $x$; similarly take $y_{j} \in f_{j}(x)$ independent of $\varepsilon$ as asserted by Henkin-equicontinuity. Then the sequence $\left(y_{j}\right)_{j>i}$ belongs to compact $f_{j}(x)$ and thus has some accumulation point $y \in f_{j}(x) \subseteq f_{i}(x)$ for each $j$ : thus yields $y \in f(x)$ independent of $\varepsilon$. W.l.o.g $y_{j} \rightarrow y$ by proceeding to a subsequence. Now let $d\left(x, x^{\prime}\right) \leq \delta$. Then by hypothesis there exists $y_{j}^{\prime} \in f_{j}\left(x^{\prime}\right)$ with $d\left(y_{j}, y_{j}^{\prime}\right) \leq \varepsilon$; and, again, an appropriate subsequence of $\left(y_{j}^{\prime}\right)$ converges to some $y^{\prime} \in f\left(x^{\prime}\right)$. Moreover, $d\left(y, y^{\prime}\right) \leq d\left(y, y_{j}\right)+d\left(y_{j}, y_{j}^{\prime}\right)+d\left(y_{j}^{\prime}, y^{\prime}\right) \leq d\left(y, y_{j}\right)+\varepsilon+d\left(y^{\prime}, y_{j}^{\prime}\right) \rightarrow \varepsilon$.

b) Consider the family $\mathcal{F}$ of all $\lambda$-continuous and pointwise compact tightenings of $f$. According to a), these form a directed complete partial order (dcpo) with respect to total restriction. More explicitly, apply Zorn's Lemma to get a maximal chain $\left(f_{i}\right), i \in I$. Then a) asserts that $g(x):=\bigcap_{i: f_{i}(x) \neq \emptyset} f_{i}(x)$ defines a $\lambda$-continuous and pointwise compact tightening of $f$. In fact a minimal one: If $h \in \mathcal{F}$ tightens $g$, then $h=f_{j}$ for some $j \in I$ because of the maximality of $\left(f_{i}\right)_{i \in I}$; hence $g$ tightens $f_{j}$.

c) A working example is $f_{n}=\overline{\left\{\left(x, x^{i}\right) \mid x \in[0,1] \wedge i \geq n\right\}}$; so that the intersection is $\{(x, 0) \mid x \in[0,1)\} \cup \cup\{(1,1)\}$.

\subsection{Relative Computability requires Henkin-Continuity}

Theorem 3.10 Let $K \subseteq \mathbb{R}$ be compact.

a) If $f: K \rightrightarrows \mathbb{R}$ is computable relative to some oracle, then it is Henkin-continuous.

b) More precisely suppose $F: \subseteq \Sigma^{\prime \omega} \rightrightarrows \Sigma^{\prime \omega}$ is a Henkin-continuous $\left(\rho_{\mathrm{sd}}, \rho_{\mathrm{sd}}\right)-$ multirealizer of $f: K \rightrightarrows \mathbb{R}$ (recall Lemma 2.4) which maps compact sets to compact sets. Then $f$ itself must be Henkin-continuous, too; and in fact has a Henkin-continuous tightening $g: K \rightrightarrows \mathbb{R}$ mapping compact sets to compact sets.

c) Conversely, if $f: K \rightrightarrows \mathbb{R}$ is Henkin-continuous and maps compact sets to compact sets, then $F:=\left.\rho_{\text {sd }}^{-1} \circ f \circ \rho_{\text {sd }}\right|^{K}$ is a Henkin-continuous $\left(\rho_{\text {sd }}, \rho_{\text {sd }}\right)-$ multirealizer of $f$ which maps compact sets to compact sets.

Proof a) Recall [28, SECTION 3] that a real relation is relatively computable iff it has a continuous $(\rho, \rho)$-realizer; equivalently [28, THEOREM 7.2.5.1]: a continuous $\left(\rho_{\text {sd }}, \rho_{\text {sd }}\right)$-realizer $F$. In particular, single-valued $F$ maps compact sets to compact sets. Moreover, $F$ is a $\left(\rho_{\text {sd }}, \rho_{\text {sd }}\right)$-multirealizer according to 
Lemma 2.4f); and has $\operatorname{dom}(F)=\operatorname{dom}\left(\left.\rho_{\mathrm{sd}}\right|^{K}\right)$ compact [28, pp.209-210], hence is even uniformly continuous, i.e. Henkin-continuous. Now apply b).

b) Proposition 3.5d) asserts $\rho_{\mathrm{sd}}^{-1}$ to be Henkin-continuous; and so is $\left(\left.\rho_{\mathrm{sd}}\right|^{K}\right)^{-1}=$ $\left.\left(\rho_{\mathrm{sd}}^{-1}\right)\right|_{K}$, compare Observation 3.4a). Now range $\left(\left(\left.\rho_{\mathrm{sd}}\right|^{K}\right)^{-1}\right)=\rho_{\mathrm{sd}}^{-1}[K]$ is compact; which $F$ maps by hypothesis to some compact set $C \subseteq \Sigma^{\prime \omega}$. Therefore $\left.\rho_{\mathrm{sd}}\right|_{C}$ is uniformly (i.e. Henkin-) continuous (Example 3.3d); and so is $\left.\rho_{\mathrm{sd}}\right|_{C} \circ$ $F \circ\left(\left.\rho_{\mathrm{sd}}\right|^{K}\right)^{-1}$ (Observation 3.4b); which, because of $C=\operatorname{range}\left(F \circ\left(\left.\rho_{\mathrm{sd}}\right|^{K}\right)^{-1}\right)$, coincides with $g:=\left.\rho_{\text {sd }} \circ F \circ \rho_{\text {sd }}^{-1}\right|_{K}$. Now this $g$ by hypothesis tightens $f$ (Lemma 2.4f); hence $f$ is also Henkin-continuous (Observation 3.4a). Moreover, $g$ maps compact sets to compact sets according to Lemma 2.4d) because each subterm $\left.\left(\rho_{\text {sd }}^{-1}\right)\right|_{K}$ [28, pp.209-210], $F$ (hypothesis), and $\rho_{\text {sd }}$ (continuous) does so.

c) Again, $\left.\rho_{\mathrm{sd}}\right|^{K}$ and $\rho_{\mathrm{sd}}^{-1}$ are Henkin-continuous by Example 3.3c) and Proposition 3.5d); hence so is the composition $F$ (Observation 3.4a). $F$ maps compact sets to compact sets according to Lemma $2.4 \mathrm{~d})$; note that $\operatorname{range}(f) \subseteq \mathbb{R}=\operatorname{dom}\left(\rho_{\text {sd }}^{-1}\right)$ and $\operatorname{range}\left(\left.\rho_{\mathrm{sd}}\right|^{K}\right)=K=\operatorname{dom}(f)$. Finally, Lemma 2.4a+b) shows $f$ to tighten $\rho_{\text {sd }} \circ F \circ \rho_{\text {sd }}^{-1}$.

\subsection{Henkin-Continuity does not imply Relative Computability}

The relation from Example 2.3c) is Henkin-continuous but not relatively computable. On the other hand, it violates the natural prerequisite of (pointwise) compactness. Instead, we modify Example 3.8 to obtain the following (counter-) example:

Example 3.11 Let

$$
\begin{aligned}
& f_{+}:=([-1 ; 0] \times\{0\}) \cup\left\{\left(x, \frac{(-1)^{n}}{n+1}\right): n \in \mathbb{N}, \frac{1}{n+1} \leq x \leq \frac{1}{n}\right\} \quad \text { and } \\
& f_{-}:=([0 ; 1] \times\{1\}) \cup\left\{\left(-x, 1+\frac{(-1)^{n}}{n+1}\right): n \in \mathbb{N}, \frac{1}{n+1} \leq x \leq \frac{1}{n}\right\} .
\end{aligned}
$$

Then $f_{1}:=f_{+} \cup f_{-}:[-1 ; 1] \rightrightarrows[-1 ; 2]$ is compact, total, and weakly 1-Lipschitz (hence Henkin-continuous), but not relatively computable; see Figure 4.

Proof Compactness and totality is readily verified. Regarding weakly 1-Lipschitz: To $x \leq 0$ set $y:=0$ and $\delta:=\varepsilon$ so that, if $x^{\prime} \leq 0, y^{\prime}:=0$ will do; and if $0<x^{\prime}<\delta$, consider $n \in \mathbb{N}$ with $1 /(n+1) \leq x^{\prime} \leq 1 / n, y^{\prime}:=(-1)^{n} /(n+1) \in f_{+}\left(x^{\prime}\right)$ has $\left|y^{\prime}-y\right|=1 /(n+1) \leq x^{\prime}<\delta=\varepsilon$. Similarly with $f_{-}$for $x \geq 0$.

It remains to show that $f_{1}$ is not relatively computable: Given a name of $x=0$, the putative realizer has the choice of producing either a name of $y_{+}=0$ or of $y_{-}=1$ : 


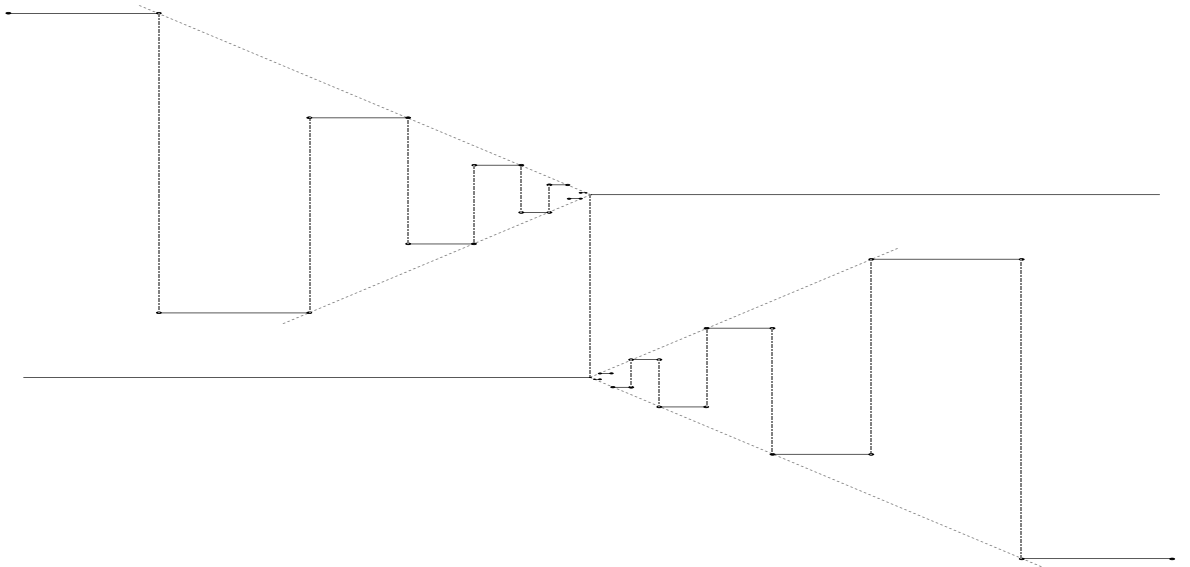

Figure 4: A compact total weakly 1-Lipschitz but not relatively computable relation. (Dashed lines indicate alignment and are not part of the graph)

knowing $x$ only up to some $\delta=1 / n, n \in \mathbb{N}$. In the first case, i.e. already tied to $f_{+}$, switch to input $x^{\prime}=1 /(n+1)$ : Then, clearly, an arbitrarily small perturbation of $x^{\prime}$ can 'fool' any algorithm computing $f_{+}$. A similar contradiction arises in the second case.

\section{Iterated Henkin-Continuity}

(Counter-)Example 3.11 suggests to strengthen Definition 3.1:

Definition 4.1 Call a total ${ }^{\dagger \dagger}$ multifunction $f: X \rightrightarrows Y$ doubly Henkin-continuous iff the following holds:

$$
\begin{aligned}
& \left(\begin{array}{cc}
\forall \varepsilon>0 & \exists \delta>0 \\
\forall x \in X & \exists y \in f(x)
\end{array}\right) \quad\left(\begin{array}{cc}
\forall \varepsilon^{\prime}>0 & \exists \delta^{\prime}>0 \\
\forall x^{\prime} \in B(x, \delta) & \exists y^{\prime} \in f\left(x^{\prime}\right) \cap B(y, \varepsilon)
\end{array}\right) \\
& \forall x^{\prime \prime} \in B\left(x^{\prime}, \delta^{\prime}\right) \exists y^{\prime \prime} \in f\left(x^{\prime \prime}\right) \cap B\left(y^{\prime}, \varepsilon^{\prime}\right) .
\end{aligned}
$$

\footnotetext{
${ }^{\dagger \dagger}$ This requirement is employed only for notational convenience and can always be met by proceeding to the restriction $\left.f\right|_{\operatorname{dom}(f)}$.
} 
Even more generally, $\ell$-fold Henkin-continuity $(\ell \in \mathbb{N})$ is to mean

$$
\begin{aligned}
& \left(\begin{array}{cc}
\forall \varepsilon_{1}>0 & \exists \delta_{1}>0 \\
\forall x_{1} \in X & \exists y_{1} \in f\left(x_{1}\right)
\end{array}\right) \quad\left(\begin{array}{cc}
\forall \varepsilon_{2}>0 & \exists \delta_{2}>0 \\
\forall x_{2} \in B\left(x_{1}, \delta_{1}\right) & \exists y_{2} \in f\left(x_{2}\right) \cap B\left(y_{1}, \varepsilon_{1}\right)
\end{array}\right) \\
& \cdots\left(\begin{array}{cc}
\forall \varepsilon_{\ell}>0 & \exists \delta_{\ell}>0 \\
\forall x_{\ell} \in B\left(x_{\ell-1}, \delta_{\ell-1}\right) & \exists y_{\ell} \in f\left(x_{\ell}\right) \cap B\left(y_{\ell-1}, \varepsilon_{\ell-1}\right)
\end{array}\right) \\
& \forall x_{\ell+1} \in B\left(x_{\ell}, \delta_{\ell}\right) \quad \exists y_{\ell+1} \in B\left(y_{\ell}, \varepsilon_{\ell}\right) \cap f\left(x_{\ell+1}\right) .
\end{aligned}
$$

Note that all occurrences of $\mathrm{Q}_{\mathrm{H}}$ are positive. Generalizing Example 3.11, we observe that this notion indeed gives rise to a proper hierarchy:

Example 4.2 To every $\ell \in \mathbb{N}$ there exists a compact total relation $f_{\ell}:[-1 ; 1] \rightrightarrows$ $[-1 ; 2]$ which is $\ell$-fold Henkin-continuous but not $(\ell+1)$-fold Henkin-continuous.

Indeed consider for instance $\ell=1$ and recall that the relation in Figure 4 is (1-fold) Henkin-continuous. Now to $x=0$ w.l.o.g. suppose $y=0$ is chosen and to $\varepsilon:=1 / 4$ some $\delta>0$. Now consider $x^{\prime}:=1 / n<\delta$ : Since $f_{+}$'jumps' at $x^{\prime}$, both choices $y^{\prime}=-(-1)^{n} / n$ and $y^{\prime}=(-1)^{n} /(n+1)$ from $f_{+}\left(x^{\prime}\right)$ contradict 2-fold Henkin-continuity for some $x^{\prime \prime}=x^{\prime} \pm \varepsilon^{\prime}$.

Figure 5 depicts an iteration $f_{2}$ of Figure 4 which, similarly, can be seen 2-fold Henkincontinuous but not 3 -fold. Repeating this iteration, one obtains a fractal sequence $f_{\ell}$ with the claimed properties.

Many properties of Henkin-continuity translate to the iterated case:

Lemma 4.3 Fix $\ell \in \mathbb{N}$.

a) If $f$ is $(\ell+1)$-fold Henkin-continuous, it is also $\ell$-fold Henkin-continuous; but not necessarily vice versa.

b) If $f: X \rightrightarrows Y$ is uniformly strongly continuous (and in particular if $f: X \rightarrow Y$ is uniformly continuous), it is $\ell$-fold Henkin-continuous for every $\ell$.

c) If $f: X \rightrightarrows Y$ is $\ell$-fold Henkin-continuous and tightens $g: \subseteq X \rightrightarrows Y$, then $g$ is $\ell$-fold Henkin-continuous (on $\operatorname{dom}(g)$ ) as well.

d) If $f: X \rightrightarrows Y$ and $g: Y \rightrightarrows Z$ are both $\ell$-fold Henkin-continuous, then so is $g \circ f$ $($ on $\operatorname{dom}(g \circ f))$.

Proof a) The first claim is obvious; failure of the converse is demonstrated in Example 4.2.

Journal of Logic \& Analysis 5:7 (2013) 


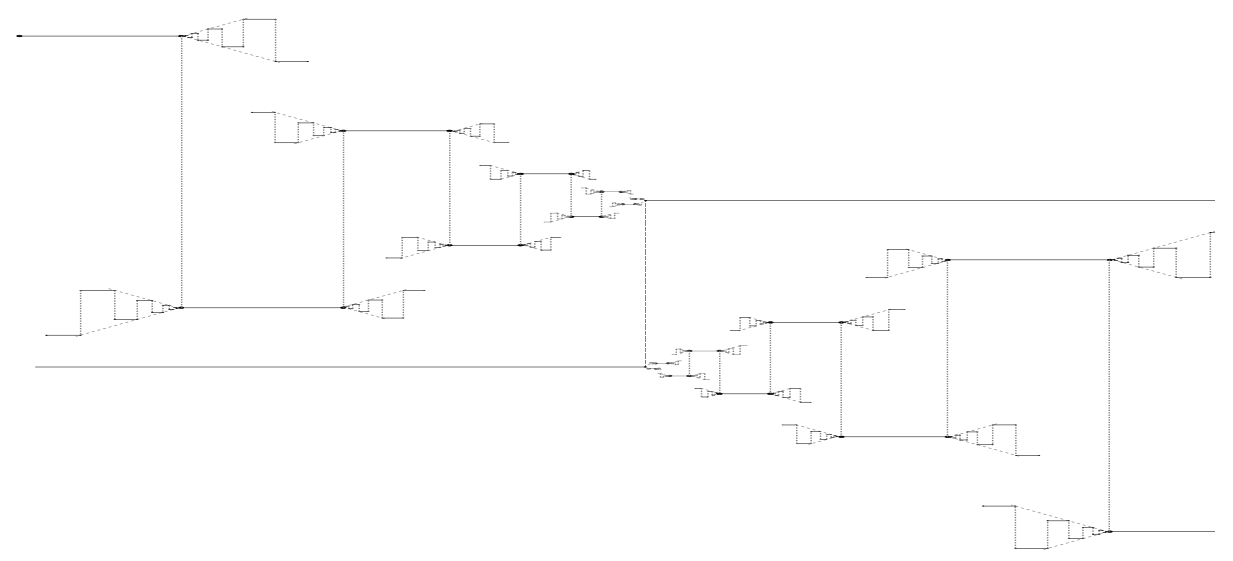

Figure 5: A compact total 2-fold, but not 3-fold, Henkin-continuous relation

b) Uniform strong continuity can be rephrased as

$$
\left(\begin{array}{cc}
\forall \varepsilon>0 & \exists \delta>0 \\
\forall x \in X & \forall y \in f(x)
\end{array}\right) \quad \forall x^{\prime} \in B(x, \delta) \quad \exists y^{\prime} \in f\left(x^{\prime}\right) \cap B(y, \varepsilon) .
$$

For a total relation this implies Henkin-continuity, where the universal quantifier is replaced by an existential one over a non-empty range since $f$ is total. Moreover, applying Equation (9) to the primed quantities in Equation (9) again yields

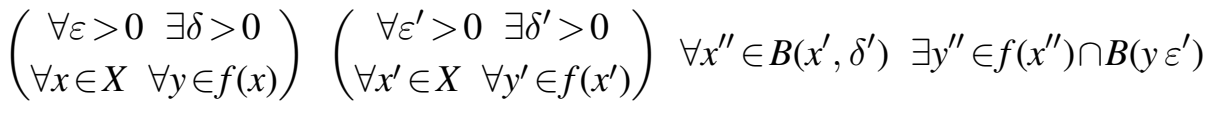
and so on inductively: which implies iterated Henkin-continuity.

c) As in the proofs of Observation 3.4a), $g$ restricts the range of the universal quantifiers occurring in Equations (8) and extends the range of the existential quantifiers.

d) By hypothesis we have Equation (8) for $f$ and the following for $g$ :

$$
\begin{aligned}
& \left(\begin{array}{cc}
\forall \delta_{1}>0 & \exists \gamma_{1}>0 \\
\forall y_{1} \in Y & \exists z_{1} \in g\left(y_{1}\right)
\end{array}\right) \quad\left(\begin{array}{cc}
\forall \delta_{2}>0 & \exists \gamma_{2}>0 \\
\forall y_{2} \in B\left(y_{1}, \gamma_{1}\right) & \exists z_{2} \in f\left(y_{2}\right) \cap B\left(z_{1}, \delta_{1}\right)
\end{array}\right) \\
& \cdots\left(\begin{array}{cc}
\forall \delta_{\ell}>0 & \exists \gamma_{\ell}>0 \\
\forall y_{\ell} \in B\left(y_{\ell-1}, \gamma_{\ell-1}\right) & \exists z_{\ell} \in f\left(y_{\ell}\right) \cap B\left(z_{\ell-1}, \delta_{\ell-1}\right)
\end{array}\right) \\
& \forall y_{\ell+1} \in B\left(y_{\ell}, \gamma_{\ell}\right) \quad \exists z_{\ell+1} \in B\left(z_{\ell}, \delta_{\ell}\right) \cap f\left(y_{\ell+1}\right) .
\end{aligned}
$$


Now inductively, to $\varepsilon_{k+1}>0$ and to $x_{k+1} \in \operatorname{dom}(g \circ f) \cap B\left(x_{k}, \delta_{k}\right)$, there exist $\delta_{k+1}>0$ independent of $x_{k+1}$ and $y_{k+1} \in f\left(x_{k+1}\right) \cap B\left(y_{k}, \varepsilon_{k}\right)$ independent of $\varepsilon_{k+1}$; to which in turn there exist $\gamma_{k+1}>0$ independent of $y_{k+1}$ and $z_{k+1} \in g\left(y_{k+1}\right) \cap B\left(z_{k}, \delta_{k}\right)$ independent of $\delta_{k}$.

\subsection{Examples and Properties}

Note that $\delta_{k}$ in Equation (8), although independent of $x_{k}$, may well depend on $x_{1}, \ldots, x_{k-1}$.

Lemma 4.4 Call $\lambda: \mathbb{N} \rightarrow \mathbb{N}$ a modulus of $\ell$-fold Henkin-continuity of $f: X \rightrightarrows Y$ if the following holds:

$$
\begin{gathered}
\forall x_{1} \in X \exists y_{1} \in f\left(x_{1}\right) \quad \forall m_{1} \in \mathbb{N} \forall x_{2} \in \bar{B}\left(x_{1}, 2^{-\lambda\left(m_{1}\right)}\right) \exists y_{2} \in f\left(x_{2}\right) \cap \bar{B}\left(y_{1}, 2^{-m_{1}}\right) \\
\text { (10) } \quad \forall m_{2}>m_{1} \quad \forall x_{3} \in \bar{B}\left(x_{2}, 2^{-\lambda\left(m_{2}\right)}\right) \quad \exists y_{3} \in f\left(x_{3}\right) \cap \bar{B}\left(y_{2}, 2^{-m_{2}}\right) \ldots \\
\ldots \quad \forall m_{\ell}>m_{\ell-1} \forall x_{\ell+1} \in \bar{B}\left(x_{\ell}, 2^{-\lambda\left(m_{\ell}\right)}\right) \exists y_{\ell+1} \in f\left(x_{\ell+1}\right) \cap \bar{B}\left(y_{\ell}, 2^{-m_{\ell}}\right) .
\end{gathered}
$$

a) If $f$ admits a modulus of $\ell$-fold Henkin-continuity, then $f$ is $\ell$-fold Henkincontinuous.

b) Consider a family $f_{i}: X_{i} \rightrightarrows Y_{i}(i \in \mathbb{N})$ of multifunctions with common modulus $\lambda$ of $\ell$-fold Henkin-continuity. Suppose the metric spaces $\left(Y_{i}, e_{i}\right)$ are uniformly bounded; w.l.o.g. all of diameter $\leq 1$. Then $\lambda+\mathrm{id}$ is a modulus of $\ell$-fold Henkin-continuity for the mapping $\prod_{i} f_{i}: \prod_{i} X_{i} \rightrightarrows \prod_{i} Y_{i}$ between product spaces with respect to the product metrics $\hat{d}\left(\left(x_{i}\right),\left(x_{i}^{\prime}\right)\right):=\sup _{i} d_{i}\left(x_{i}, x_{i}^{\prime}\right) / 2^{i}$ and $\hat{e}\left(\left(y_{i}\right),\left(y_{i}^{\prime}\right)\right)=\sup _{i} e_{i}\left(y_{i}, y_{i}^{\prime}\right) / 2^{i}$.

c) For $f$ single-valued, $\lambda$ is a modulus of $\ell$-fold Henkin-continuity iff it is a modulus of uniform continuity in the usual sense. If $\lambda$ is a modulus of $\ell$-fold Henkin-continuity to $f: \subseteq X \rightrightarrows Y$, then it is also one of any loosening of $f$. Moreover suppose $\mu$ is a modulus of $\ell$-fold Henkin-continuity to $g: \subseteq Y \rightrightarrows Z$; then $\lambda \circ \mu$ is a modulus of $\ell$-fold Henkin-continuity to $g \circ f: \subseteq X \rightrightarrows Z$.

The mapping $\prod_{i} f_{i}$ amounts to 'parallelization' in the sense of BRATTKA and GHERARDI [6]. Note $\left(\prod_{i} f_{i}\right)^{-1}=\prod_{i} f_{i}^{-1}$.

Proof a) In Equation (8) inductively assign $\left(\varepsilon_{1}, \ldots, \varepsilon_{k}\right)$ to $\delta_{k}:=2^{-\lambda\left(m_{k}\right)}$ for

$$
m_{k}:=\left\lceil k+\log _{2} 1 / \min \left\{\varepsilon_{1}, \ldots, \varepsilon_{k}\right\}\right\rceil
$$

according to Equation (10); and simultaneously assign $x_{1}$ to $y_{1}$ as well as inductively $x_{k+1} \in B\left(x_{k}, \delta_{k}\right) \subseteq \bar{B}\left(x_{k}, 2^{-m_{k}}\right)$ to $y_{k+1} \in f\left(x_{k+1}\right) \cap \bar{B}\left(y_{k}, 2^{-m_{k}}\right) \subseteq$ $B\left(y, \varepsilon_{k}\right)$ according to Equation (10). 
b) We demonstrate the case $\ell=2$; the general case proceeds analogously. To $\bar{x}=$ $\left(x_{i}\right)_{i} \in \prod_{i} X_{i}$ assign $\bar{y}=\left(y_{i}\right)_{i} \in \prod Y_{i}$ with $y_{i} \in f_{i}\left(x_{i}\right)$ according to the hypothesis. Now given $m \in \mathbb{N}, \sup _{i} d_{i}\left(x_{i}, x_{i}^{\prime}\right) / 2^{i}=\hat{d}\left(\bar{x}, \bar{x}^{\prime}\right) \leq 2^{-\lambda(m)-m}$ implies $d_{i}\left(x_{i}, x_{i}^{\prime}\right) \leq$ $2^{-\lambda(m)}$ for $i \leq m$; hence there exist by hypothesis $y_{i}^{\prime} \in f_{i}\left(x_{i}^{\prime}\right) \cap \bar{B}\left(y_{i}, 2^{-m}\right)$, $i \leq m$; and totality of $f_{i}$ yields $y_{i}^{\prime} \in f_{i}\left(x_{i}^{\prime}\right)$ also for $i>m$; and $e\left(y_{i}^{\prime}, y_{i}\right) \leq 1$ by the uniform boundedness hypothesis: in either case, $e\left(y_{i}^{\prime}, y_{i}\right) / 2^{i} \leq 2^{-m}$ for all $i: \hat{e}\left(\bar{y}^{\prime}, \bar{y}\right) \leq 2^{-m}$. Similarly, given $m^{\prime} \in \mathbb{N}, \hat{d}\left(\bar{x}^{\prime}, \bar{x}^{\prime \prime}\right) \leq 2^{-\lambda\left(m^{\prime}\right)-m^{\prime}}$ implies $d_{i}\left(x_{i}^{\prime}, x_{i}^{\prime \prime}\right) \leq 2^{-\lambda\left(m^{\prime}\right)}$ for $i \leq m^{\prime}$ and yields $y_{i}^{\prime \prime} \in f_{i}\left(x_{i}^{\prime \prime}\right) \cap \bar{B}\left(y_{i}^{\prime}, 2^{-m^{\prime}}\right)$; while totality of $f_{i}$ yields $y_{i}^{\prime \prime} \in f_{i}\left(x_{i}^{\prime \prime}\right)$ also for $i>m$ : together with boundedness, $\hat{e}\left(\bar{y}^{\prime \prime}, \bar{y}^{\prime}\right) \leq 2^{-m^{\prime}}$.

c) immediate.

We now strengthen Proposition 3.5c)+d) and Theorem 3.10:

Proposition 4.5 a) Let $x=\sum_{n=-N}^{\infty} a_{n} 2^{-n}$ be a signed digit expansion and $k \in$ $\mathbb{N}$ such that $\left(a_{n}, a_{n+1}\right) \in\{10, \overline{1} 0,01,0 \overline{1}, 00\}$ for each $n>k$. Then every $x^{\prime} \in \bar{B}\left(x, 2^{-k} / \mathbf{6}\right)$ admits a signed digit expansion $x^{\prime}=\sum_{n=-N}^{\infty} b_{n} 2^{-n}$ satisfying $a_{n}=b_{n} \forall n \leq k$ and $\left(b_{n}, b_{n+1}\right) \in\{10, \overline{1} 0,01,0 \overline{1}, 00\}$ for all $n>k+\mathbf{1}$.

b) The inverse $\rho_{\mathrm{sd}}^{-1}$ of the signed-digit representation has $\lambda: m \mapsto m+2$ as modulus of $\ell$-fold Henkin-continuity, independent of $\ell$.

c) If $f: K \rightrightarrows \mathbb{R}$ is computable relative to some oracle, then it is $\ell$-fold Henkincontinuous.

More precisely if $F: \subseteq \Sigma^{\prime \omega} \rightrightarrows \Sigma^{\prime \omega}$ is an $\ell$-fold Henkin-continuous $\left(\rho_{\mathrm{sd}}, \rho_{\mathrm{sd}}\right)-$ multirealizer of $f: K \rightrightarrows \mathbb{R}$ which maps compact sets to compact sets, then $f$ itself must be $\ell$-fold Henkin-continuous, too; and in fact has an $\ell$-fold Henkincontinuous tightening $g: K \rightrightarrows \mathbb{R}$ mapping compact sets to compact sets. Conversely, if $f: K \rightrightarrows \mathbb{R}$ is $\ell$-fold Henkin-continuous and maps compact sets to compact sets, then $F:=\left.\rho_{\mathrm{sd}}^{-1} \circ f \circ \rho_{\mathrm{sd}}\right|^{K}$ is an $\ell$-fold Henkin-continuous $\left(\rho_{\mathrm{sd}}, \rho_{\mathrm{sd}}\right)$-multirealizer of $f$ which maps compact sets to compact sets.

Proof a) First consider the case $a_{k+1}=0$. Then $x^{\prime \prime}:=\sum_{n=-N}^{k} a_{n} 2^{-n}=$ $\sum_{n=-N}^{k+1} a_{n} 2^{-n}$ has $\left|x-x^{\prime \prime}\right| \leq 2^{-k} / 3$ due to Proposition 3.5b). Hence $x^{\prime}-x^{\prime \prime}=$ $\left(x^{\prime}-x\right)+\left(x-x^{\prime \prime}\right) \in\left[-2^{-k} / 2 ; 2^{-k} / 2\right] \subseteq\left[-\frac{2}{3} \cdot 2^{-k} ;+\frac{2}{3} \cdot 2^{-k}\right]$ has, again according to Proposition 3.5b), a signed digit expansion $x^{\prime}-x^{\prime \prime}=\sum_{n=k+1}^{\infty} b_{n} 2^{-n}$ with $\left(b_{n}, b_{n+1}\right) \in\{10, \overline{1} 0,01,0 \overline{1}, 00\}$ for all $n$. This yields $x^{\prime}=\left(x^{\prime}-x^{\prime \prime}\right)+x^{\prime \prime}=$ $\sum_{n=-N}^{k} a_{n} 2^{-n}+\sum_{n=k+1}^{\infty} b_{n} 2^{-n}$ an expansion with the claimed properties.

It remains to consider the case $a_{k+1}=1$ (and $a_{k+1}=\overline{1}$ proceeds analogously). Here the hypothesis on $\left(a_{n}, a_{n+1}\right)$ asserts $a_{k+2}=0$. Therefore 
$x^{\prime \prime}:=\sum_{n=-N}^{k+\mathbf{1}} a_{n} 2^{-n}=\sum_{n=-N}^{k+2} a_{n} 2^{-n}$ has $\left|x-x^{\prime \prime}\right| \leq 2^{-k} / 6$ due to Proposition 3.5b). Hence $x^{\prime}-x^{\prime \prime}=\left(x^{\prime}-x\right)+\left(x-x^{\prime \prime}\right) \in\left[-\frac{2}{3} \cdot 2^{-(k+1)} ;+\frac{2}{3} \cdot 2^{-(k+1)}\right]$ has, again according to Proposition 3.5b), a signed digit expansion $x^{\prime}-x^{\prime \prime}=$ $\sum_{n=k+2}^{\infty} b_{n} 2^{-n}$ with $\left(b_{n}, b_{n+1}\right) \in\{10, \overline{1} 0,01,0 \overline{1}, 00\}$ for all $n$. This yields $x^{\prime}=\left(x^{\prime}-x^{\prime \prime}\right)+x^{\prime \prime}=\sum_{n=-N}^{k+1} a_{n} 2^{-n}+\sum_{n=k+2}^{\infty} b_{n} 2^{-n}$ an expansion with the claimed properties.

b) For $k=0,1,2, \ldots$ let $\mathcal{D}_{k}$ denote the set

$$
\left\{\bar{\sigma} \in \operatorname{dom}\left(\rho_{\mathrm{sd}}\right): \sigma_{N}=^{\prime} .^{\prime}, \quad\left(\sigma_{n}, \sigma_{n+1}\right) \in\{10, \overline{1} 0,01,0 \overline{1}, 00\} \forall n>N+k\right\}
$$

Then a) yields the following property $(*)$

$\forall x \in \mathbb{R} \forall \bar{\sigma} \in \rho_{\text {sd }}^{-1}(x) \cap \mathcal{D}_{k} \forall x^{\prime} \in \bar{B}\left(x, 2^{-k-3}\right) \exists \bar{\sigma}^{\prime} \in \rho_{\text {sd }}^{-1}\left(x^{\prime}\right) \cap \bar{B}\left(\bar{\sigma}, 2^{-k-1}\right) \cap \mathcal{D}_{k+1}$

Indeed a signed digit expansion $\sum_{n=-N}^{\infty} a_{n} 2^{-n}$ corresponds to a $\rho_{\mathrm{sd}}-$ name $\left(a_{-N}, \cdots, a_{0}, ., a_{1}, \ldots\right)$ with the dot at position $N \geq 0$; hence expansions coinciding up to index $k$ correspond to names coinciding up to index $N+k$, i.e., with distance $\leq 2^{-N-k-1}$.

We now apply Equation $\left(^{*}\right)$ once with $k:=m_{1}-1$ and $x:=x_{1}$ and $x^{\prime}:=x_{2}$, in order to conclude (weakening " $\forall \bar{\sigma}$ " to " $\exists \bar{\sigma}$ " since $\mathcal{D}_{0} \subseteq \mathcal{D}_{k}$ and $\rho_{\text {sd }}\left(\mathcal{D}_{0}\right)=\mathbb{R}$ according to Proposition 3.5a) that $\lambda: m \mapsto m+2$ is a modulus of one-fold Henkin-continuity:

$\forall x_{1} \in \mathbb{R} \exists \bar{\sigma}^{(1)} \in \rho_{\text {sd }}^{-1}\left(x_{1}\right) \quad \forall x_{2} \in \bar{B}\left(x, 2^{-\left(m_{1}+2\right)}\right) \exists \bar{\sigma}^{(2)} \in \rho_{\text {sd }}^{-1}\left(x_{2}\right) \cap \bar{B}\left(\bar{\sigma}, 2^{m_{1}}\right) \cap \mathcal{D}_{m_{1}}$

Moreover, to $m_{2}>m_{1}$, apply Equation (*) again with $x:=x_{2}$ and $k:=m_{2}-1 \geq$ $m_{1}$ (hence $\mathcal{D}_{m_{1}} \subseteq \mathcal{D}_{k}$ ) and $\bar{\sigma}:=\bar{\sigma}^{(2)}$ and $x_{3}:=x^{\prime}$ to continue, concluding that $\lambda$ is a modulus of two-fold Henkin-continuity:

$$
\forall x_{3} \in \bar{B}\left(x, 2^{-\left(m_{2}+2\right)}\right) \exists \bar{\sigma}^{(3)} \in \rho_{\text {sd }}^{-1}\left(x_{3}\right) \cap \bar{B}\left(\bar{\sigma}, 2^{-m_{2}}\right) \cap \mathcal{D}_{m_{2}}
$$

and so on.

c) Similarly to the proof of Theorem 3.10 but with b) and Lemma 4.3 in place of Proposition 3.5d) and Observation 3.4. 


\subsection{Infinitary Henkin-continuity and the Main Result}

In view of Example 4.2, let us strengthen Definition 4.1 further to the case $\ell=\omega$. The idea is to call $f: X \rightrightarrows Y \omega$-fold Henkin-continuous if is satisfies

$$
\begin{array}{r}
\left(\begin{array}{cc}
\forall \varepsilon_{1}>0 & \exists \delta_{1}>0 \\
\forall x_{1} \in X & \exists y_{1} \in f\left(x_{1}\right)
\end{array}\right)\left(\begin{array}{cc}
\forall \varepsilon_{2}>0 & \exists \delta_{2}>0 \\
\forall x_{2} \in B\left(x_{1}, \delta_{1}\right) & \exists y_{2} \in f\left(x_{2}\right) \cap B\left(y_{1}, \varepsilon_{1}\right)
\end{array}\right) \\
\cdots\left(\begin{array}{cc}
\forall \varepsilon_{\ell}>0 & \exists \delta_{\ell}>0 \\
\forall x_{\ell} \in B\left(x_{\ell-1}, \delta_{\ell-1}\right) & \exists y_{\ell} \in f\left(x_{\ell}\right) \cap B\left(y_{\ell-1}, \varepsilon_{\ell-1}\right)
\end{array}\right) \\
\left(\begin{array}{cc}
\forall \varepsilon_{\ell+1}>0 & \exists \delta_{\ell+1}>0 \\
\forall x_{\ell} \in B\left(x_{\ell}, \delta_{\ell}\right) & \exists y_{\ell+1} \in f\left(x_{\ell+1}\right) \cap B\left(y_{\ell}, \varepsilon_{\ell}\right)
\end{array}\right) \cdots
\end{array}
$$

Note that a relation might well be $\ell$-fold Henkin-continuous for every $\ell \in \mathbb{N}$ without being $\omega$-fold Henkin-continuous: For the former, $\delta_{1}$ is permitted to depend on $\left(\varepsilon_{1}\right.$ and on) $\ell$ ! Of course assigning a semantics to infinitary Henkin-logic in general is touchy, particularly in view of (lack of) determinacy. On the other hand, the intuitive notion intended as above can be rephrased as the existence of a relation

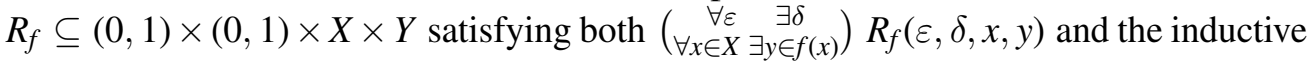
property

$\forall \varepsilon, \delta, x, y: \quad R_{f}(\varepsilon, \delta, x, y) \Rightarrow\left(\begin{array}{cc}\forall \varepsilon^{\prime} & \exists \delta^{\prime} \\ \forall x^{\prime} \in B(x, \delta) & \exists y^{\prime} \in f\left(x^{\prime}\right) \cap B(y, \varepsilon)\end{array}\right) R_{f}\left(\varepsilon^{\prime}, \delta^{\prime}, x^{\prime}, y^{\prime}\right)$

— which suggests the following

Definition 4.6 Call $f: X \rightrightarrows Y \omega$-fold Henkin-continuous if the greatest fixedpoint $R_{f}$ (according to Knaster-Tarski) of the monotonously decreasing operator

$$
R^{\prime} \mapsto\left\{(\varepsilon, \delta, x, y) \in R^{\prime}:\left(\begin{array}{c}
\forall \varepsilon^{\prime} \quad \exists \delta^{\prime} \\
\forall x^{\prime} \in B(x, \delta) \exists y^{\prime} \in f\left(x^{\prime}\right) \cap B(y, \varepsilon)
\end{array}\right) R^{\prime}\left(\varepsilon^{\prime}, \delta^{\prime}, x^{\prime}, y^{\prime}\right)\right\}
$$

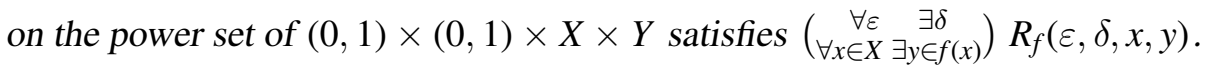

Again, $\omega$-fold Henkin-continuity generalizes uniform continuity of single-valued functions and is closed both under tightening and under composition. Lemma 4.4a) translates into Item a) of the following

Proposition $4.7 \quad$ a) If total $f: X \rightrightarrows Y$ is pointwise compact and admits a modulus $\lambda: \mathbb{N} \rightarrow \mathbb{N}$ of $\ell$-fold Henkin-continuity independent of $\ell$, then $f$ is $\omega$-fold Henkin-continuous. 
b) Suppose $F: \subseteq \Sigma^{\omega} \rightrightarrows \Sigma^{\omega}$ maps compact sets to compact sets and is $\omega$-fold Henkincontinuous. Then $F$ admits a continuous total selection $G: \operatorname{dom}(F) \rightarrow \Sigma^{\omega}$.

c) Moreover if $\lambda$ is a modulus of $\ell$-fold Henkin-continuity of $F$ for every $\ell$, then $m \mapsto \lambda(m+1)+m$ is a modulus of uniform continuity of $G$.

Item $b$ ) shows that $\omega$-fold Henkin-continuity may be regarded as a uniform version of König's Lemma; compare KoHLENBACH [14]. The proof of items b+c) employs the following slight generalization of [28, DEFINITION 2.1.10+LEMMA 2.1.11.2].

Observation 4.8 Call a partial function $h: \subseteq \Sigma^{*} \rightarrow \Sigma^{*}$ monotone if $h(\vec{u}) \sqsubseteq h(\vec{v})$ whenever $\vec{u} \sqsubseteq \vec{v}$ and $\vec{u}, \vec{v} \in \operatorname{dom}(h)$. Here “ $\vec{u} \sqsubseteq \vec{v}$ " means that $\vec{u} \in \Sigma^{*}$ is an initial segment of $\vec{v} \in \Sigma^{*}$; formally: $\exists \vec{w} \in \Sigma^{*}: \vec{u} \circ \vec{w}=\vec{v}$. Similarly, for $\bar{v} \in\{0,1\}^{\omega}$ write " $\vec{u} \sqsubseteq \bar{v}$ " if $\bar{v}=\vec{u} \circ \bar{w}$ for some $\bar{w} \in\{0,1\}^{\omega}$. For $\left.\bar{\sigma} \in\{0,1\}\right\}^{\omega}$ abbreviate $\left.\bar{\sigma}\right|_{\leq n}:=\left(\sigma_{1}, \ldots, \sigma_{n}\right) \in\{0,1\}^{n}$. Call $h$ unbounded on $\bar{\sigma}$ if its length $|h|$ is unbounded on $\left\{\left.\bar{\sigma}\right|_{\leq n}: n \in \mathbb{N}\right\} \cap \operatorname{dom}(h)$.

a) Every monotone partial $h: \subseteq \Sigma^{*} \rightarrow \Sigma^{*}$ gives rise to a continuous partial function $h_{\omega}: \subseteq \Sigma^{\omega} \rightarrow \Sigma^{\omega}$ with $\operatorname{dom}\left(h_{\omega}\right)=\{\bar{v}: h$ unbounded on $\bar{v}\}$ via $\bar{v} \mapsto \sup _{n} h\left(\left.\bar{v}\right|_{\leq n}\right)$.

b) Under the additional hypothesis that $h\left[\Sigma^{\mu(\ell)}\right] \subseteq \Sigma^{\geq \ell}$ for some $\mu: \mathbb{N} \rightarrow \mathbb{N}, h_{\omega}$ is uniformly continuous with modulus $\mu$.

Proof of Proposition 4.7 a) First, similarly to the proof of Lemma 4.4, inductively assign $\left(\varepsilon_{1}, x_{1}, \ldots, x_{k-1}, \varepsilon_{k}\right)$ to $\delta_{k}:=2^{-\lambda\left(m_{k}\right)}$ for

$$
m_{k}:=\left\lceil k+\log _{2} 1 / \min \left\{\varepsilon_{1} / 2, \ldots, \varepsilon_{k} / 2\right\}\right\rceil>m_{k-1}
$$

according to Equation (10). It remains to assign $\left(x_{1}, \varepsilon_{1}, \ldots, \varepsilon_{k-1}, x_{k}\right)$ to some $y_{k}$ as follows: For $k=1$, the hypothesis provides a family of assignments $x_{1} \mapsto y_{1, \ell} \in f\left(x_{1}\right)$ for every $\ell$, each one extending to inductive assignments $\left(x_{2}, \ldots, x_{k}\right) \mapsto y_{k, \ell} \in f\left(x_{k}\right) \cap \bar{B}\left(y_{\ell, k-1}, 2^{-m_{k-1}}\right)$ for $\quad x_{k} \in \bar{B}\left(x_{k-1}, 2^{-\lambda\left(m_{k-1}\right)}\right)$,

$2 \leq k \leq \ell+1$. Since $f$ is supposed to be pointwise compact, $\left(y_{1, \ell}\right)_{\ell}$ has some accumulation point $y_{1} \in f\left(x_{1}\right)$; hence, for infinitely many $\ell, e\left(y_{1}, y_{1, \ell}\right) \leq$ $\left.2^{-m_{1}}\right) \leq \varepsilon_{1} / 2$; and to each such $\ell$, the hypothesis provides assignments

$$
\bar{B}\left(x_{1}, 2^{-\lambda\left(m_{1}\right)}\right) \ni x_{2} \mapsto y_{2, \ell} \in f\left(x_{2}\right) \cap \bar{B}\left(y_{1, \ell}, 2^{-m_{1}}\right) \subseteq \bar{B}\left(y_{1}, \varepsilon_{1}\right) .
$$

Now, again by pointwise compactness, these $y_{2, \ell} \in f\left(x_{2}\right)$ have an accumulation point $y_{2} \in f\left(x_{2}\right) \cap \bar{B}\left(y_{1}, \varepsilon_{1}\right)$ belonging to $\bar{B}\left(y_{2, \ell}, 2^{-m_{2}}\right)$ for infinitely many of the 
(infinitely many) $\ell$ considered so far; and to each such $\ell$, the hypothesis provides assignments

$$
\bar{B}\left(x_{2}, 2^{-\lambda\left(m_{2}\right)}\right) \ni x_{3} \mapsto y_{3, \ell} \in f\left(x_{3}\right) \cap \bar{B}\left(y_{2, \ell}, 2^{-m_{2}}\right) \subseteq \bar{B}\left(y_{2}, \varepsilon_{2}\right)
$$

and so on inductively.

b) Note that the triangle inequality in $\Sigma^{\omega}$ strengthens to $d(\bar{x}, \bar{z}) \leq \max \{d(\bar{x}, \bar{y}), d(\bar{y}, \bar{z})\}$. We now construct a monotone partial $h$ according to Observation 4.8, prove it unbounded on $\operatorname{dom}(F)$, and that $G:=h_{\omega}$ tightens $F$. To this end define sets $h^{-1}[\vec{y}]$ by induction on $|\vec{y}|=n$ as follows:

For $n=1$ let $\varepsilon_{1}:=2^{-1}$ and consider $\delta_{1}>0$ provided by the hypothesis; w.l.o.g. $\delta_{1} \geq 2^{-n_{1}}$ for some $n_{1} \in \mathbb{N}$. Then to each $\vec{x}_{1} \in \Sigma^{m}$ choose some $\bar{x}_{1} \in \operatorname{dom}(F)$ having $\vec{x}_{1}$ as initial segment (if such a $\bar{x}_{1}$ exists) and define $h\left(\vec{x}_{1}\right):=\left.\bar{y}_{1}\right|_{\leq 1}$ for $\bar{y}_{1} \in F\left(\bar{x}_{1}\right)$ as provided by the hypothesis; $h\left(\vec{x}_{1}\right):=\perp$ if $\vec{x}_{1} \circ \Sigma^{\omega} \cap \operatorname{dom}(F)=\emptyset$. Next let $\varepsilon_{2}:=2^{-2}$ and, for each $\vec{x}_{1} \in \Sigma^{n_{1}}$ with $h\left(\vec{x}_{1}\right)$ defined, consider $\delta_{2} \geq: 2^{-n_{2}}$ provided by the hypothesis and choose, to every $\vec{x}_{2} \in \vec{x}_{1} \circ \sum^{n_{2}-n_{1}}$, some $\bar{x}_{2} \in \operatorname{dom}(F) \cap\left(\vec{x}_{2} \circ \Sigma^{\omega}\right)$ (where possible) to define $h\left(\vec{x}_{2}\right):=\left.\bar{y}_{2}\right|_{\leq 2}$ where $\bar{y}_{2} \in F\left(\bar{x}_{2}\right)$ is provided by the hypothesis; if $\vec{x}_{2} \circ \Sigma^{\omega} \cap \operatorname{dom}(F)=\emptyset$, then we choose $h\left(\vec{x}_{2}\right):=h\left(\vec{x}_{1}\right)$ where $x_{1}$ is the prefix of $x_{2}$ dealt with in the previous stage. And so on inductively, let $\varepsilon_{\ell}:=2^{-\ell}$ and, for each $\vec{x}_{\ell-1} \in \Sigma^{n_{\ell-1}}$ with $h\left(\vec{x}_{\ell-1}\right)$ defined, consider $\delta_{\ell} \geq: 2^{-n_{\ell}}$ provided by the hypothesis and choose, to every $\vec{x}_{\ell} \in \vec{x}_{\ell-1} \circ \Sigma^{n_{\ell}-n_{\ell-1}}$, some $\bar{x}_{\ell} \in \operatorname{dom}(F) \cap\left(\vec{x}_{\ell} \circ \Sigma^{\omega}\right)$ to define $h\left(\vec{x}_{\ell}\right):=\left.\bar{y}_{\ell}\right|_{\leq \ell}$ where $\bar{y}_{\ell} \in F\left(\bar{x}_{\ell}\right)$ is provided by the hypothesis; $h\left(\vec{x}_{\ell}\right):=h\left(\vec{x}_{\ell-1}\right)$ if $\vec{x}_{\ell} \circ \Sigma^{\omega} \cap \operatorname{dom}(F)=\emptyset$.

The thus defined partial $h$ is monotone indeed: If $h\left(\vec{x}_{\ell}\right)=\perp$, then $\emptyset \neq$ $\left(\vec{x}_{\ell} \circ \Sigma^{\omega}\right) \cap \operatorname{dom}(F)$ and hence $\emptyset=\left(\vec{x}_{\ell+1} \circ \Sigma^{\omega}\right) \cap \operatorname{dom}(F)$; i.e. $h\left(\vec{x}_{\ell} \circ \Sigma^{*}\right)=\perp$. On the other hand for $h\left(\vec{x}_{\ell}\right)=\left.\bar{y}_{\ell}\right|_{\leq \ell}$ and $h\left(\vec{x}_{\ell+1}\right)=\left.\bar{y}_{\ell+1}\right|_{\leq \ell+1}, \vec{x}_{\ell+1} \in \vec{x}_{\ell} \circ \sum^{n_{\ell+1}-n_{\ell}}$ implies $\bar{x}_{\ell+1} \in \vec{x}_{\ell+1} \circ \Sigma^{\omega}$, i.e. $\bar{x}_{\ell+1} \in \bar{B}\left(\bar{x}_{\ell}, 2^{-n_{\ell}}\right)$; hence $\bar{y}_{\ell+1} \in \bar{B}\left(\bar{y}_{\ell}, 2^{-\ell}\right)$ : $h\left(\vec{x}_{\ell}\right) \sqsubseteq h\left(\vec{x}_{\ell+1}\right)$. Moreover $h$ is unbounded on $\bar{x} \in \operatorname{dom}(F)$ : For every $\ell$, $\vec{x}_{\ell} \sqsubseteq \bar{x}$ implies $\bar{x} \in\left(\vec{x}_{\ell} \circ \Sigma^{\omega}\right) \cap \operatorname{dom}(F) \neq \emptyset$ and $\left|h\left(\vec{x}_{\ell}\right)\right|=\ell$. Finally, by construction, $h_{\omega}(\bar{x})=\lim _{\ell} \bar{y}_{\ell}$ for $\bar{y}_{\ell} \in F\left(\bar{x}_{\ell}\right)$ with $\bar{x}_{\ell} \in \bar{B}\left(\bar{x}, 2^{-n_{\ell-1}}\right)$. Since $\left\{\bar{x}_{\ell}: \ell\right\} \cup\{\bar{x}\} \subseteq \operatorname{dom}(F)$ is compact, $F$ by hypothesis maps it to a compact set containing $\left\{\bar{y}_{\ell}: \ell\right\}$ and thus also $\lim _{\ell} \bar{y}_{\ell}=\bar{y} \in F(\bar{x}): h_{\omega}$ is a selection of $F$.

c) In the proof of $\mathrm{b}), \delta_{\ell}=2^{-n_{\ell}}$ could depend on $\left(\varepsilon_{\ell}=2^{-\ell}\right.$ and on) $\bar{x}_{\ell-1}$. But if $\lambda$ is a modulus of $\ell$-fold Henkin-continuity, the proof of a) yields $\delta_{\ell}=2^{-\lambda(\ell+1)-\ell}$ independent of $\bar{x}_{\ell-1}$. Hence the thus constructed $h$ satisfies the hypothesis of Observation 4.8b).

Given a metric space $\mathbf{X}=(X, d)$, w.l.o.g. with $d$ bounded, we denote by $\widehat{\mathbf{X}}$ the metric 
space obtained by equipping the set $X^{\mathbb{N}}$ with the metric $\hat{d}(\mathbf{x}, \mathbf{y})=\sup _{i \in \mathbb{N}} d(\mathbf{x}(i), \mathbf{y}(i)) / 2^{i}$ from Lemma 4.4b). This metric is chosen such that the topology induced on $\widehat{\mathbf{X}}$ coincides with the product topology derived from $\mathbf{X}$. For $f: \mathbf{X} \rightrightarrows \mathbf{Y}$, define $\widehat{f}: \widehat{\mathbf{X}} \rightrightarrows \widehat{\mathbf{Y}}$ component-wise via $\widehat{f}(\mathbf{x})(i)=f(\mathbf{x}(i))$.

Fact 4.9 a) Every separable metric space is homeomorphic to a subspace of $\widehat{[0 ; 1]}$, the Hilbert Cube.

b) Every separable metric space $\mathbf{X}$ with dense sequence $\left(x_{1}, x_{2}, \ldots\right)=\bar{x}$ gives rise to a canonical admissible representation $\alpha: \subseteq \Sigma^{\omega} \rightarrow X$.

c) If $\mathbf{X}$ is compact, the above $\alpha$ can be chosen as total and in particular proper.

d) Any two admissible representations of the same metric space are computably equivalent relative to some oracle.

e) Let $\mathbb{N} \times \mathbb{N} \ni(n, m) \mapsto\langle n, m\rangle:=(n+m-2) \cdot(n+m-1) / 2+n \in \mathbb{N}$ denote CANTOR's integer pairing function. Then the string pairing function

$$
\widehat{\Sigma^{\omega}} \rightarrow \Sigma^{\omega}, \quad\left(\sigma_{n, m}\right)_{n, m \in \omega} \mapsto\left(\tau_{k}\right)_{k \in \omega} \quad \text { with } \quad \tau_{\langle n, m\rangle}=\sigma_{n, m}
$$

and its inverse $\imath$ are both uniformly continuous.

For a) refer, e.g. to MoRRIS [20, THEOREM 9.4.11]. Claim b) is from [28, DEF. 8.1.2+THEOREM 8.1.4]; and c) follows from BRATTKA, DE BRECHT, and PAUly [4, ProposiTION 4.1]. For d) combine [28, THEOREMS 2.3.8.1+3.2.8.1] with the observation that any $h: \Sigma^{*} \rightarrow \Sigma^{*}$ can be encoded into some oracle.

In the sequel abbreviate $\widetilde{\rho_{\text {sd }}}:=\widehat{\rho_{\text {sd }}} \circ \imath: \subseteq \Sigma^{\prime \omega} \rightarrow \widehat{[0 ; 1]}$. Our main theorem now strengthens Theorem 3.10 and Proposition 4.5 to $\omega$-fold Henkin-continuity on arbitrary compact separable metric spaces and establishes a converse:

Theorem 4.10 Let $\mathbf{Y}$ be a separable metric spaces with representation $\beta$ according to Fact $4.9 b)$ and $\mathbf{X}$ in addition compact with $\alpha$ according to Fact 4.9c).

a) The inverse $\rho_{\mathrm{sd}}^{-1}$ of the signed-digit representation for real numbers is $\omega$-fold Henkin-continuous; and so is the inverse ${\widetilde{\rho_{\mathrm{sd}}}}^{-1}$ of the admissible representation of the Hilbert cube. Restricted to compact subsets of Cantor space, every admissible representation of a metric space is $\omega$-fold Henkin-continuous.

b) Let $f: \mathbf{X} \rightrightarrows \mathbf{Y}$ be relatively $(\alpha, \beta)$-computable. Then there exists $g: \mathbf{X} \rightrightarrows \mathbf{Y}$ tightening $f$ which is relatively computable and maps compact sets to compact sets. 
c) If $f: \mathbf{X} \rightrightarrows \mathbf{Y}$ is $(\alpha, \beta)$-computable relative to some oracle, it is $\omega$-fold Henkin-continuous.

d) Suppose $f: \mathbf{X} \rightrightarrows \mathbf{Y}$ maps compact sets to compact sets and is $\omega$-fold Henkincontinuous. Then $f$ is $(\alpha, \beta)$-computable relative to some oracle.

This provides the desired topological characterization of relative computability:

Corollary 4.11 For $\mathbf{X}, \mathbf{Y}$ separable and $\mathbf{X}$ compact, a total relation $f: X \rightrightarrows Y$ mapping compact sets to compact sets (and in particular one with compact graph) is computable relative to some oracle iff it is $\omega$-fold Henkin-continuous.

More generally suppose $\mathbf{X}$ is locally compact and $f: \mathbf{X} \rightrightarrows \mathbf{Y}$ maps compact sets to compact sets. Then $f$ is relatively computable iff it is locally $\omega$-fold Henkin-continuous in the sense that every $x \in \mathbf{X}$ has a compact neighborhood $K$ such that $\left.f\right|_{K}$ is $\omega$-fold Henkin-continuous.

Indeed the countably many oracle algorithms computing $\left.f\right|_{K}$ for a basis of compact sets $K$ can be combined into one single oracle.

Proof of Theorem 4.10 a) The first claim follows from Proposition 4.5b+c) and Proposition 4.7a). For the second claim, combine Fact 4.9e) with Lemma 4.4b+c), observing that $[0 ; 1]$ is bounded. Concerning the third claim, an admissible representation is continuous; and thus uniformly continuous on compact sets: now apply Lemma 4.4c).

b) By hypothesis $\alpha: \Sigma^{\omega} \rightarrow X$ is proper, that is, $\alpha^{-1}: X \rightarrow \Sigma^{\omega}$ maps compact sets to compact sets. Let $F: \Sigma^{\omega} \rightarrow \Sigma^{\omega}$ be a relatively computable and thus continuous $(\alpha, \beta)$-realizer of $f$. As both $F$ and $\beta$ are continuous single-valued functions, they map compact sets to compact sets. By Lemma $2.4 \mathrm{~d}+\mathrm{f}$ ) we find $g=\beta \circ F \circ \alpha^{-1}$ to tighten $f$ as desired.

c) As a consequence of Fact 4.9a+d), we may regard $X$ as a compact subset of the Hilbert cube $\widehat{[0 ; 1]}$. By a), $\alpha^{-1}=\left.{\widetilde{\rho_{\mathrm{sd}}}}^{-1}\right|_{X}$ is $\omega$-fold Henkin-continuous. According to $\mathrm{b}$ ) and Lemma 4.3c) we may w.l.o.g. suppose that $f$ maps compact sets to compact sets and in particular that $C:=f[X]$ is compact. A continuous $(\alpha, \beta)$-realizer $F: \subseteq \Sigma^{\omega} \rightarrow \Sigma^{\omega}$ of $f$ has $\operatorname{dom}(F)=\alpha^{-1}[\operatorname{dom}(f)]={\widetilde{\rho_{\mathrm{sd}}}}^{-1}[X]$ compact; hence is (uniformly and) $\omega$-fold Henkin-continuous; and so is $\left.\beta\right|_{C} \rightarrow Y$. Moreover $\left.\beta\right|_{C} \circ F \circ \alpha^{-1}$ tightens $f$ (Lemma 2.4f); hence is $\omega$-fold Henkincontinuous, and so is $f$. 
d) Now we also regard compact $K:=f[X] \subseteq Y$ as a subspace of $\widehat{[0 ; 1]}$ and w.l.o.g. (Fact 4.9d) suppose $\beta=\left.\widetilde{\rho_{\mathrm{sd}}}\right|^{K}$. Again $\beta^{-1}=\left.\widetilde{\rho_{\mathrm{sd}}}{ }^{-1}\right|_{K}$ is $\omega$-fold Henkin-continuous and maps compact sets to compact sets; same for continuous $\alpha$ on $\operatorname{dom}(\alpha)={\widetilde{\rho_{\mathrm{sd}}}}^{-1}[X]$ compact; and thus also $F:=\beta^{-1} \circ f \circ \alpha$. According to Proposition 4.7b), $F$ therefore admits a continuous selection $G$ on $\operatorname{dom}(F)=$ $\operatorname{dom}(\alpha)$ : a relatively computable $(\alpha, \beta)$-realizer of $f$.

\section{Conclusion}

We have proposed a hierarchy of notions of uniform continuity for real relations based on the Henkin quantifier; and shown its $\omega$-th level to characterize relative computability.

This extends the well-known characterization of uniform continuity in terms of relative computability for (single-valued real) functions - and justifies considering the intrinsic (i.e. computer-science free) definition of $\omega$-fold Henkin-continuity as 'right' generalization.

The proof takes a 'detour' from arbitrary compact metric spaces via the Hilbert cube equipped with the parallelization of the signed-digit representation; which makes one wonder about a more direct argument.

Our condition may be considered descriptionally simpler than the previous characterization from [7]: Although both notions do leave the first-order framework, Definition 4.6 employs fixedpoint logic whereas Fact 1.1 existentially quantifies over subsets of $\mathbb{R}$. As a 'bonus' it yields a quantitative refinement in terms of relativized computational complexity in Section 6 below.

Remark 5.1 A proof that some relation $f$ (such as the multivalued sign from the introduction) is uncomputable usually proceeds by 'fooling' a hypothetical Turing machine $\mathcal{M}$ : First run it on (specific approximations to) some argument $x_{0} \in \operatorname{dom}(f)$ and obtain (approximations up to some finite precision to) a value $y_{0} \in f\left(x_{0}\right)$; then restart $\mathcal{M}$ and feed to it the finite information read in the first run - but now serving as approximations to some $x_{1} \neq x_{0}$ where the approximations produced previously (and, deterministically, again) disagree with every $y_{1} \in f\left(x_{1}\right)$.

In view of this (meta-) observation it seems worthwhile pointing out that the proofs to Example 2.6a) and Example 3.11 involve two rounds: A hypothetical machine is first run on some $x_{0}$ to obtain a finite approximation to some $y_{0} \in f\left(x_{0}\right)$; then re-run on some $x_{1}$ close to $x_{0}$ to obtain a finite approximation to some $y_{1} \in f\left(x_{1}\right)$; and finally again on some $x_{2}$ close to $x_{1}$ to finally arrive at a contradiction. 
Similarly, showing the relation $f_{\ell}$ from Example 4.2 uncomputable involves $\ell+1$ re-runs in order to 'fool' any hypothetical oracle machine. On the other hand $f_{\ell}$ does become computable when permitting the machine one so-called mind change: a concept originating in learning theory [9] that has recently received increasing interest in real computability [31, 4] and proof theoy [15].

Acknowledgements: The presentation of this work has benefited from many helpful remarks by a very careful anonymous reviewer. The first author thanks ANUJ DAWAR for an introduction to logical expressiveness. The last author is grateful to ULRICH KOHLENBACH for pointing out that already M.J. BEESON had observed the relevance of the Henkin quantifier to continuity in constructive mathematics; and to KLAUS WEIHRAUCH for providing the 'right' notion of composition for relations. MARTIN OTто has provided great advice on fixedpoint logic. This work was supported in parts by the Royal Society International Exchange Grant IE111233, by the Marie Curie International Research Staff Exchange Scheme Fellowship 294962 within the 7th European Community Framework Programme, and by the German Research Foundation (DFG) project Zi 1009/4.

\section{Bonus: Topological Characterization of Relatively Poly- time Computable Relations}

While every computable real function must be continuous, a quantitative complexitytheoretic refinement states that any polynomial-time computable $f:[0 ; 1] \rightarrow \mathbb{R}$ must have a polynomial modulus of uniform continuity, see Ko [13, THEOREM 2.19] and compare [28, EXERCiSE 7.1.7]. This has led KaWAMURA, MüLler, RÖSNICK, and ZIEGLER [11, EXAMPLE 1.12] to the following explicit

Example 6.1 The function

$$
f:[0 ; 1] \rightarrow \mathbb{R}, \quad 0 \mapsto 0, \quad 0<x \mapsto 1 / \ln (e / x)
$$

is computable in exponential time but, lacking a polynomial modulus of uniform continuity, not in polynomial time - and oracles do not help.

Here, $f:[0 ; 1] \rightarrow \mathbb{R}$ is considered computable in time $t(n)$ if a Turing machine can, given a sequence $a_{m} / 2^{m+1}$ of dyadic approximations to any $x \in[0 ; 1]$ up to error $2^{-m}$, output dyadic approximations $b_{n} / 2^{n+1}$ to $f(x)$ up to error $2^{-n}$ such that the $n$-th 
approximation appears within $t(n)$ steps independent of $x$. Modulo polynomial time (shorter: polytime) equivalence, dyadic approximations may be replaced by (initial segments of) a signed digit expansion [28, THEOREM 9.4.3].

Definition 6.2 a) A partial function $F: \subseteq \Sigma^{\omega} \rightarrow \Sigma^{\omega}$ is computable in time $t: \mathbb{N} \rightarrow \mathbb{N}$ if a Type-2 Machine can, given $\bar{\sigma} \in \operatorname{dom}(F)$, produce $\bar{\tau}=F(\bar{\sigma})$ such that the $n$-th symbol of $\bar{\tau}$ appears within $t(n)$ steps.

b) For spaces $X$ and $Y$ with representations $\alpha$ and $\beta$, a partial multivalued mapping $f: \subseteq X \rightrightarrows Y$ is computable in time $t(n)$ if it admits an $(\alpha, \beta)$-realizer $F: \subseteq \Sigma^{\omega} \rightarrow \Sigma^{\omega}$ computable in time $t(n)$.

Of course computably equivalent representations may lead to different notions under the refined view of complexity, some even become useless [28, EXAMPLE 7.2.1]. (Meta-) Conditions that avoid such pitfalls are devised for instance in [29, 25]. We now characterize polytime computability relative to some oracle, first for functions on Cantor space:

Lemma 6.3 For a function $F: \subseteq \Sigma^{\omega} \rightarrow \Sigma^{\omega}$, the following are equivalent:

i) There exists an oracle relative to which $F$ is computable in polytime.

ii) $F$ has a polynomially bounded modulus of uniform continuity.

iii) There exists a monotone partial $h: \subseteq \Sigma^{*} \rightarrow \Sigma^{*}$ with $h\left[\Sigma^{p(\ell)}\right] \subseteq \Sigma^{\geq \ell}$ for some polynomial $p$ such that $\left.h\right|_{\omega}$ (according to Observation 4.8) extends $F$.

Proof i) $\Rightarrow$ ii) Within time $p(n)$, a Type-2 Machine can read at most $p(n)$ symbols of the input stream before producing the $n$-th output symbol - and oracles do not help.

ii) $\Rightarrow$ iii) Suppose $d(F(\bar{\sigma}), F(\bar{\tau})) \leq 2^{-n}$ for $d(\bar{\sigma}, \bar{\tau}) \leq 2^{-p(n)}, \bar{\sigma}, \bar{\tau} \in \operatorname{dom}(F)$. Put differently: For every $\vec{x} \in \Sigma^{p(n)}$ there exists some $\vec{y} \in \Sigma^{n}$ such that $F\left[\vec{x} \circ \Sigma^{\omega}\right] \subseteq$ $\vec{y} \circ \Sigma^{\omega}$. Then the inductive construction in [28, p.37 paragraph 5] yields $h$ with the desired properties.

iii) $\Rightarrow$ i) Encode $h$ into oracle $\mathcal{O}_{h}:=\operatorname{graph}(h)=\{\langle\vec{x}, \vec{y}\rangle: h(\vec{x})=\vec{y}\}$. Now given $\bar{\sigma} \in \operatorname{dom}(F)$ and $n \in \mathbb{N}$, search for some (unique) $y_{1} \in \Sigma$ with $\left\langle\bar{\sigma}_{\leq p(1)}, y_{1}\right\rangle \in \mathcal{O}_{h}$; then for some $y_{2}$ with $\left\langle\bar{\sigma}_{\leq p(2)}, y_{1} y_{2}\right\rangle \in \mathcal{O}_{h}$, and so on inductively to obtain $\vec{y} \in \Sigma^{n}$ with $\left\langle\bar{\sigma}_{\leq p(n)}, \vec{y}\right\rangle \in \mathcal{O}_{h}$ : This evaluates $F(\bar{\sigma})$ up to error $2^{-n}$.

Theorem 6.4 a) The inverse $\widetilde{\rho_{\mathrm{sd}}}-1$ of the representation of the Hilbert cube has a polynomial modulus of $\ell$-fold Henkin-continuity independent of $\ell$; and so does $\widetilde{\rho_{\mathrm{sd}}}$, restricted to compact subsets (either of the image or of the domain). 
b) Fix compact $\hat{K} \subseteq \widehat{[0 ; 1]}$ and total $f: \hat{K} \rightrightarrows \widehat{[0 ; 1]}$. If $f$ is polytime $\left(\widetilde{\rho_{\text {sd }}}, \widetilde{\rho_{\text {sd }}}\right)-$ computable relative to some oracle, it has a tightening mapping compact sets to compact sets that admits a polynomial modulus $\lambda: \mathbb{N} \rightarrow \mathbb{N}$ of $\ell$-fold Henkin-continuity independent of $\ell$.

c) Fix compact $\hat{K} \subseteq \widehat{[0 ; 1]}$ and total $f: \hat{K} \rightrightarrows \widehat{[0 ; 1]}$ mapping compact sets to compact sets and admitting polynomial modulus $\lambda: \mathbb{N} \rightarrow \mathbb{N}$ of $\ell$-fold Henkincontinuity independent of $\ell$. Then $f$ is polytime $\left(\widetilde{\rho_{\mathrm{sd}}}, \widetilde{\rho_{\mathrm{sd}}}\right)$-computable relative to some oracle.

d) Fix compact $K \subseteq[0 ; 1]^{d}$ and identify it with $\hat{K}:=K \times\{0\}^{\omega} \subseteq \widehat{[0 ; 1]}$. Then the representations $\left.\rho_{\text {sd }}^{d}\right|^{K}$ and $\left.\widetilde{\rho_{\mathrm{sd}}}\right|^{\hat{K}}$ are mutually polytime equivalent in the sense that $K \ni \vec{x} \mapsto \vec{x} \times \overline{0} \in \hat{K}$ is $\left(\rho_{\text {sd }}^{d}, \widetilde{\rho_{\text {sd }}}\right)$-computable in polytime and $\hat{K} \ni \vec{x} \times \overline{0} \mapsto \vec{x} \in K$ is $\left(\widetilde{\rho_{\mathrm{sd}}}, \rho_{\mathrm{sd}}^{d}\right)$-computable in polytime.

Corollary 6.5 For $K \subseteq \mathbb{R}^{d}$ compact, a total $f: K \rightrightarrows \mathbb{R}^{e}$ mapping compact sets to compact sets is polytime computable relative to some oracle iff it admits a polynomial modulus of $\ell$-fold Henkin-continuity independent of $\ell$.

Proof of Theorem 6.4 a) We record that Cantor's integer pairing function from Fact $4.9 \mathrm{e}$ ) and its inverse are classically polytime computable with respect to arguments and values encoded in unary. It follows that the induced string pairing function $\imath: \widehat{\Sigma^{\prime \omega}} \rightarrow \Sigma^{\prime \omega}$ and its inverse both have a polynomial modulus of uniform continuity. By Proposition 4.5b) and Lemma $4.4 \mathrm{~b}+\mathrm{c}$ ), also ${\widetilde{\rho_{\mathrm{sd}}}}^{-1}=\imath^{-1} \circ \widehat{\rho_{\mathrm{sd}}}$ has a polynomial modulus of $\ell$-fold Henkin-continuity independent of $\ell$. Concerning $\widetilde{\rho_{\mathrm{sd}}}$, combine Example 3.3c) with Example 2.5b) and Lemma 4.4c).

b) If $F$ is a relatively polytime-computable $\left(\widetilde{\rho_{\mathrm{sd}}}, \widetilde{\rho_{\mathrm{sd}}}\right)$-realizer of $f$, then it has a polynomial modulus of $\ell$-fold Henkin-continuity independent of $\ell$ (Lemmas $6.3+4.4 \mathrm{c})$; and $\left(\left.\widetilde{\rho_{\mathrm{sd}}}\right|^{\hat{K}}\right)^{-1}$ has one according to a). Now $\left(\widetilde{\rho_{\mathrm{sd}}}\right)^{-1}[\hat{K}]$ is compact; and mapped by $F$ to some compact set $C \subseteq \Sigma^{\prime \omega}$. Therefore $\left.\widetilde{\rho_{\mathrm{sd}}}\right|_{C}$ has a polynomial modulus of $\ell$-fold Henkin-continuity independent of $\ell$ by a). Together we conclude that $\left.\widetilde{\rho_{\text {sd }}}\right|_{C} \circ F \circ\left(\left.\widetilde{\rho_{\text {sd }}}\right|^{\hat{K}}\right)^{-1}$ has a polynomial modulus of $\ell$-fold Henkin-continuity independent of $\ell$ and tightens $f$.

c) Suppose $f$ admits a polynomial modulus of $\ell$-fold Henkin-continuity independent of $\ell$. Then so does $F:=\left.\left(\widetilde{\rho_{\mathrm{sd}}}\right)^{-1} \circ f \circ \widetilde{\rho_{\mathrm{sd}}}\right|^{\hat{K}}$ according to a) and Lemma $\left.4.4 \mathrm{~b}+\mathrm{c}\right)$. And $F$ maps compact sets to compact sets (Lemma 2.4d). Hence $F$ has a selection $G$ on $\operatorname{dom}(F)=\operatorname{dom}\left(\left.\rho_{\text {sd }}^{d}\right|^{\hat{K}}\right)$ with polynomial modulus of uniform continuity (Proposition 4.7c). This is relatively polytime computable due to Lemma 6.3. 
d) Given a $d$-tuple of $\rho_{\mathrm{sd}}$-names $\bar{\sigma}^{(1)}, \ldots, \bar{\sigma}^{(d)}$ of $x_{1}, \ldots, x_{d}$ it is easy to extend it with a $\rho_{\mathrm{sd}}$-name $\bar{\sigma}^{(0)}$ of 0 and to combine these, using the Cantor pairing function, into a $\widetilde{\rho_{\mathrm{sd}}}-$ name of $\left(x_{1}, \ldots, x_{d}, 0,0, \ldots\right)$; and vice versa.

\section{References}

[1] J. BARWISE: Some Applications of Henkin quantifiers, Israel Journal of Mathematics 25 (1976), 47-63; doi:10.1007/BF02756560.

[2] V. BRATTKA: Recursive characterization of computable real-valued functions and relations, Theoretical Computer Science 162 (1996), 45-77; doi:10.1016/03043975(95)00249-9.

[3] V. BRATTKA: Computability over topological structures, in Computability and Models (S.B.Cooper and S.S. Goncharov, Eds), Springer, 2003, 93-136; doi:10.1007/978-14615-0755-0_5.

[4] V. Brattka, M. De Brecht, A. Pauly: Closed choice and a Uniform Low Basis Theorem, Annals of Pure and Applied Logic 163:8 (2012), 968-1008; doi:10.1016/j.apal.2011.12.020.

[5] M.J. BeESON: Foundations of Constructive Mathematics, Springer Series of Modern Surveys in Mathematics, 3:6, 1985.

[6] V. BRATTKA, G. GHERARDI: Weihrauch degrees, omniscience principles and weak computability, Bulletin of Symbolic Logic 17:4 (2011), 73-117; doi:10.2178/js1/1294170993.

[7] V. BRATTKa, P. Hertling: Continuity and computability of relations, Informatik Berichte FernUniversität in Hagen, 164 (1994).

[8] V. BrattKa, M. Ziegler: Computability in linear algebra, Theoretical Computer Science 326 (2004), 187-211; doi:10.1016/j.tcs.2004.06.022.

[9] M. DE BRECHT, A. YAMAMOTO: Mind change complexity of inferring unbounded unions of pattern languages from positive data, Theoretical Computer Science 411 (2010), 976-985; doi:10.1007/11894841_13.

[10] V. GREGORIADES: The descriptive set-theoretic complexity of the set of points of discontinuity of a multi-valued function, Logical Methods in Computer Science 7:4 (2011); doi:10.2168/LMCS-7(4:2)2011.

[11] A. Kawamura, N.T. Müller, C. RöSNick, M. Ziegler: Parameterized uniform complexity in numerics: from smooth to analytic, from $\mathcal{N} \mathcal{P}$-hard to polytime, arXiv: 1211.4974 (2012).

[12] E. Klein, A.C. Thompson: Theory of Correspondences, Including Appplications to Mathematical Economics, Canadian Mathematical Society Series of Monographs and Advanced Texts, Wiley, 1984. 
[13] K.-I. Ko: Computational Complexity of Real Functions, Birkhäuser, 1991.

[14] U. KoHLENBACH: On uniform weak König's lemma, Annals of Pure and Applied Logic 114 (2002), 103-116; doi:10.1016/S0168-0072(01)00077-X.

[15] U. KoHLEnBach, P. SAFARIK: Fluctuations, effective learnability and metastability in analysis, to appear in Annals of Pure and Applied Logic; doi:10.1016/j.apal.2013.07.014.

[16] C. Kreitz, K. WeIHRAUCH: Compactness in constructive analysis revisited, Annals of Pure and Applied Logic 36 (1987), 29-38; doi:10.1016/0168-0072(87)90010-8.

[17] M. Krynicki, M. Mostowski: Henkin Quantifiers, in Quantifiers: Logics, Models and Computation, Kluwer Academic Publishers I, 1995, 193-262; doi:10.1007/978-94-0170522-6_1.

[18] H. LUCKHARDT: A fundamental effect in computations on real numbers, Theoretical Computer Science 5 (1977), 321-324; doi:10.1016/0304-3975(77)90048-2.

[19] J. MILLER: Degrees of unsolvability of continuous functions, Journal of Symbolic Logic 69:2 (2004), 555-584; doi:10.2178/js1/1082418543.

[20] S.A. MoRRIS: Topology Without Tears, http://topologywithouttears .net, 2012.

[21] A. PAULY: How incomputable is finding Nash equilibria?, Journal of Universal Computer Science 16:18 (2010), 2686-2710; doi:10.3217/jucs-016-18-2686.

[22] A. PAULY: A new introduction to the theory of represented spaces, arXiv:1204.3763, 2013.

[23] P. SCHOdL, A. NeUMAIER: Continuity notions for multi-valued mappings with possibly disconnected images, Reliable Computing 16 (2012), 84-101.

[24] M. SCHRÖDER: Extended admissibility, Theoretical Computer Science 284:2 (2002), 519-538; doi:10.1016/S0304-3975(01)00109-8.

[25] M. SCHRÖDER: Spaces allowing type-2 complexity theory revisited, Mathematical Logic Quarterly 50 (2004), 443-459; doi:10.1002/malq.200310111.

[26] D. SpREEN: Effectivity and effective continuity of multifunctions, The Journal of Symbolic Logic 75:2 (2010), 602-640; doi:10.2178/jsl/1268917496.

[27] J. VÄÄNÄNEN: Dependence Logic: A New Approach to Independence Friendly Logic, Cambridge University Press, 2007.

[28] K. WeIHRAUCH: Computable Analysis, Springer, 2000.

[29] K. WeIHRAUCH: Computational complexity on computable metric spaces, Mathematical Logic Quarterly 49:1 (2003), 3-21; doi:10.1002/malq.200310001.

[30] K. WeIHRAUCH: The computable multi-functions on multi-represented sets are closed under programming, Journal of Universal Computer Science 14:6 (2008), 801-844; doi:10.3217/jucs-014-06-0801. 
[31] M. ZIEGLER: Revising type-2 computation and degrees of discontinuity, in Proceedings of the Third International Conference on Computability and Complexity in Analysis,(D. Cenzer, R. Dillhage, T. Grubba, K. Weihrauch eds.), Elsevier Electronic Notes in Theoretical Computer Science, 167 (2007); doi:10.1016/j.entcs.2006.08.015.

[32] M. ZIEGLER: Real computation with least discrete advice: a complexity theory of nonuniform computability, Annals of Pure and Applied Logic 163:8 (2012), 1108-1139; doi:10.1016/j.apal.2011.12.030.

Cambridge University

Technische Universität Darmstadt

amp66@cam.ac.uk, ziegler@mathematik.tu-darmstadt.de

http://www.cl.cam.ac.uk/ amp66, http://www.mathematik.tu-darmstadt.de/ ziegler

Received: 6 June 2012 Revised: 22 July 2013 